\title{
GLOBALIZATION OF GROUP COHOMOLOGY IN THE SENSE OF ALVARES-ALVES-REDONDO
}

\author{
MIKHAILO DOKUCHAEV, MYKOLA KHRYPCHENKO, AND JUAN JACOBO SIMÓN
}

\begin{abstract}
Recently E. R. Alvares, M. M. Alves and M. J. Redondo introduced a cohomology for a group $G$ with values in a module over the partial group algebra $K_{\mathrm{par}}(G)$, which is different from the partial group cohomology defined earlier by the first two named authors of the present paper. Given a unital partial action $\alpha$ of $G$ on a (unital) algebra $\mathcal{A}$ we consider $\mathcal{A}$ as a $K_{\text {par }}(G)$-module in a natural way and study the globalization problem for the cohomology in the sense of Alvares-Alves-Redondo with values in $\mathcal{A}$. The problem is reduced to an extendibility property of cocycles. Furthermore, assuming that $\mathcal{A}$ is a product of blocks, we prove that any cocycle is globalizable, and globalizations of cohomologous cocycles are also cohomologous. As a consequence we obtain that the Alvares-Alves-Redondo cohomology group $H_{\text {par }}^{n}(G, \mathcal{A})$ is isomorphic to the usual cohomology group $H^{n}(G, \mathcal{M}(\mathcal{B}))$, where $\mathcal{M}(\mathcal{B})$ is the multiplier algebra of $\mathcal{B}$ and $\mathcal{B}$ is the algebra under the enveloping action of $\alpha$.
\end{abstract}

\section{INTRODUCTION}

In algebra 2-cocycles of groups appeared as factor sets in the study of group extensions, in the theory of projective group representations, and in the construction of crossed products. Also 1-cocycles were already hidden in Kummers' result, widely known as Hilbert's Theorem 90. Exel's idea of $C^{*}$-crossed products by twisted partial group actions 24 stimulated the purely algebraic treatment of the notion in [10, the foundation of the theory of partial projective group representations in 17, [18, 19] and 22, as well as the definition and study of group cohomology based on partial actions in [13] and related extensions in [14] and [15].

A $G$-module over a group $G$ is an action of $G$ on an abelian group, and the starting point in 13 is the replacement of a $G$-module by a partial $G$-module. The latter means a unital partial action of $G$ on a commutative monoid. This fits nicely the concept of a twisted partial group action in Exel's crossed products and the notion of the equivalence of twisted partial actions from [11]. The partial group cohomology of [13] found applications to partial projective representations [13, [22], to the construction of a Chase-Harrison-Rosenberg type seven terms exact sequence [20], 21, associated to a partial Galois extension of commutative rings [12], and to the study of ideals of (global) reduced $C^{*}$-crossed products in [27. It also inspired the treatment of partial cohomology from the point of view of Hopf algebras [4.

2010 Mathematics Subject Classification. Primary 20J06; Secondary 16W22, 18 G60.

Key words and phrases. Partial action, cohomology, globalization. 
Observe that the term "partial $G$-module", where $G$ is a group, is being understood in two related but different senses. Initially it was used under the name of a "partial $G$-space" in 23] when dealing with partial group representations. The latter are closely related to partial actions (see [6]), and they are governed by the partial group algebra $K_{\mathrm{par}}(G)$, whose purely algebraic version was introduced in $[9]$. So a partial $G$-space in [23] means a $K_{\text {par }}(G)$-module, or equivalently, a $K$-module $M$ equipped with a partial representation $G \rightarrow \operatorname{End}_{K}(M)$. This is a natural point of view and it was adopted in [2] giving an alternative approach to partial cohomology, with an appropriate notion of a trivial partial $G$-module. Despite the two "partial cohomology" theories can be defined using rather similar formulas, there is a significant difference between them due to the fact that the category of $K_{\text {par }}(G)$ modules is abelian, whereas the category of partial $G$-modules from [13] is not even additive. A natural situation in which the two cohomologies can be compared is the case of a $K_{\text {par }}(G)$-module structure which comes from a unital partial action of $G$ on a commutative ring, and a relation between the two approaches occurs only for 0-cohomology.

The present paper deals mainly with the globalization problem of the cohomology from [2. The technique worked out in [11] to deal with the globalization problem of twisted partial group actions on rings was further developed in 16 to obtain a globalization result for the partial group cohomology in the sense of [13, where the partial $G$-module is not only a commutative semigroup but also a commutative unital ring, which is a direct product of indecomposable rings (blocks). It turns out, that our globalization technique can also be applied to the cohomology from [2], assuming, that the base $K_{\mathrm{par}}(G)$-module comes from a partial action of $G$ on a product of (not necessarily commutative) blocks.

We begin by recalling some background in Section 1, and in Subsection 2.1 we point out in Proposition 2.1 the only direct relation we found between the cohomologies in 13 and 2 . In the subsequent sections we deal only with cohomology in the sense of [2]. Given a $K_{\mathrm{par}}(G)$-module $M$, the cohomology groups from [2] with values in $M$ are denoted by $H_{\text {par }}^{n}(G, M)$. Partial derivations were introduced in [2] as $K$-linear maps $\delta: K_{\text {par }}(G) \rightarrow M$ satisfying a certain Leibniz rule, and the cohomology group $H_{\text {par }}^{1}(G, M)$ was characterized as the quotient group of the partial derivations by the subgroup of the principal partial derivations. In Subsection 2.2 we correct an overview in the definition of a partial derivation given in [2] and offer one more interpretation of the elements of $H_{p a r}^{1}(G, M)$ which involves some maps $f: G \rightarrow M$ satisfying a kind of 1-cocycle identity (see Theorem 2.6). The commutative subalgebra $B$ of $K_{\mathrm{par}}(G)$, defined in $\left[8\right.$ in order to endow $K_{\mathrm{par}}(G)$ with the structure of a partial crossed product, can be naturally seen as a $K_{\mathrm{par}}(G)$-module, which plays the role of trivial module in the definition of the cohomology in 22. In Subsection 2.3 we construct a projective resolution of the $K_{\text {par }}(G)$-module $B$ adapting some ideas of the free resolution from [13. Theorem 2.21 asserts that our projective resolution gives the cohomology from [2. Independently, Dessislava Kochloukova also produced a projective resolution of $B$ exploring the crossed product structure of $K_{\mathrm{par}}(G)$. Dessislava's notes were the starting point for a collaboration which resulted in the preprint [3]. 
In Subsection 3.1 we begin our work with the globalization problem. Given a unital partial action of $G$ on a (not necessarily commutative) algebra $\mathcal{A}$ over a (commutative) ring $K$, we obtain in a standard way a $K_{\text {par }}(G)$-module structure on $\mathcal{A}$ and study the globalization problem for the cohomology with values in such a module. As a first step, we prove in Theorem 3.6 that a cocycle $w \in Z_{p a r}^{n}(G, \mathcal{A})$ is globalizable if and only if there exists a certain extension $\widetilde{w}: G^{n} \rightarrow \mathcal{A}$ of $w$ which satisfies a "more global" $n$-cocycle equality. Our global (usual) cocycles take values in the additive group of the multiplier algebra $\mathcal{M}(\mathcal{B})$ of $\mathcal{B}$ where $\mathcal{B}$ is the algebra under the global action $\beta$ of $G$, which is an enveloping action of $\alpha$ (i.e. a globalization of $\alpha$ with a certain minimality condition). In order to construct $\widetilde{w}$, we assume, as in [10] and [16], that $\mathcal{A}$ is a product of blocks. Our main technical work is done in Subsection 3.2 in which for an arbitrary cocycle $w \in Z_{p a r}^{n}(G, \mathcal{A})$ we construct an $n$-cocycle $w^{\prime} \in Z_{p a r}^{n}(G, \mathcal{A})$ cohomologous to $w$ and suitable for a desired extension $\widetilde{w^{\prime}}$ (see Theorem 3.17). In Subsection 3.3 we use $\widetilde{w^{\prime}}$ to produce an extension $\widetilde{w}$ of $w$ needed for the application of Theorem 3.6. This leads to Theorem 3.20 which asserts that any cocycle from $Z_{\text {par }}^{n}(G, \mathcal{A})$ is globalizable. Our uniqueness result is Theorem 3.22 which says that globalizations of cohomologous $n$-cocycles from $Z_{p a r}^{n}(G, \mathcal{A})$ are also cohomologous. The two latter facts imply our final result Corollary 3.23 which states that $H_{\text {par }}^{n}(G, \mathcal{A})$ is isomorphic to the classical cohomology group $H^{n}(G, \mathcal{M}(\mathcal{B}))$.

\section{Preliminaries}

In this section we recall some basic notions and facts used in the sequel. Our algebras will be over a commutative unital ring $K$. A partial action $\alpha$ of a group $G$ on a non-necessarily unital algebra (or a ring) $\mathcal{A}$ is a family of two-sided ideals $\mathcal{D}_{g}$ of $\mathcal{A}$ and algebra (or ring) isomorphisms $\left\{\alpha_{g}: \mathcal{D}_{g^{-1}} \rightarrow \mathcal{D}_{g} \mid g \in G\right\}$ such that

(i) $\alpha_{1}=\operatorname{id}_{\mathcal{A}}$,

(ii) $\exists \alpha_{h}(a), \exists \alpha_{g}\left(\alpha_{h}(a)\right) \Rightarrow \exists \alpha_{g h}(a)$ and $\alpha_{g}\left(\alpha_{h}(a)\right)=\alpha_{g h}(a)$.

Here $\exists \alpha_{g}(a)$ means that $a \in \mathcal{D}_{g^{-1}}$.

Replacing above the word "algebra" by "semigroup" we obtain the concept of a partial action of $G$ on a semigroup. A partial action $\alpha$ is called unital if each $\mathcal{D}_{g}$ is unital, i.e. $\mathcal{D}_{g}=1_{g} \mathcal{A}$, where $1_{g}$ is a central idempotent of $\mathcal{A}$. If we replace "algebra" by "set", "ideal" by "subset" and "isomorphism" by "bijection", we come to the notion of a partial action of $G$ on an abstract set.

Let $\alpha=\left\{\alpha_{g}: \mathcal{D}_{g^{-1}} \rightarrow \mathcal{D}_{g} \mid g \in G\right\}$ and $\alpha^{\prime}=\left\{\alpha_{g}^{\prime}: \mathcal{D}_{g^{-1}}^{\prime} \rightarrow \mathcal{D}_{g}^{\prime} \mid g \in G\right\}$ be partial actions of a group $G$ on algebras $\mathcal{A}$ and $\mathcal{A}^{\prime}$, respectively. Recall from [1, 18] that a morphism of partial actions $(\mathcal{A}, \alpha) \rightarrow\left(\mathcal{A}^{\prime}, \alpha^{\prime}\right)$ is a homomorphism of algebras $\varphi: \mathcal{A} \rightarrow \mathcal{A}^{\prime}$ such that $\varphi\left(\mathcal{D}_{x}\right) \subseteq \mathcal{D}_{x}^{\prime}$ and $\varphi \circ \alpha_{x}=\alpha_{x}^{\prime} \circ \varphi$ on $\mathcal{D}_{x^{-1}}$. Thus, partial actions of a group $G$ on algebras (rings, semigroups or sets) form a category 1

The partial group cohomology theory, as developed in [13], is based on the concept of a unital partial $G$-module, which generally means a unital partial action of $G$ on a commutative monoid. On the other hand, the cohomology theory introduced more recently in [2] deals with (usual) modules over $K_{\text {par }}(G)$. Recall that the partial group algebra $K_{\text {par }}(G)$ of a group $G$ over a commutative ring $K$ can be seen

\footnotetext{
${ }^{1}$ Notice that isomorphic partial actions were called equivalent in 8 .
} 
as the semigroup algebra $K \mathcal{S}(G)$, where $\mathcal{S}(G)$ is the monoid defined by R. Exel in 25. for an arbitrary group $G$ by means of generators $\{[g] \mid g \in G\}$ and relations:

$$
\begin{aligned}
{\left[g^{-1}\right][g][h] } & =\left[g^{-1}\right][g h], \\
{[g][h]\left[h^{-1}\right] } & =[g h]\left[h^{-1}\right], \\
{[g]\left[1_{G}\right] } & =[g],
\end{aligned}
$$

where $g, h \in G$ (consequently, $\left[1_{G}\right][g]=[g]$ ). The elements $e_{g}=[g]\left[g^{-1}\right]$ are commuting idempotents in $\mathcal{S}(G)$, and one easily obtains the useful relations

$$
[g] e_{h}=e_{g h}[g], e_{h}[g]=[g] e_{g^{-1} h},
$$

for all $g, h \in G$. According to [25, Proposition 2.5], each element $s \in \mathcal{S}(G)$ can be written as

$$
s=e_{h_{1}} \ldots e_{h_{k}}[g],
$$

for some $k \in \mathbb{N}, h_{1}, \ldots, h_{k}, g \in G$. One can assume that

(i) $h_{i} \neq h_{j}$ for $i \neq j$,

(ii) $h_{i} \neq g$ and $h_{i} \neq 1_{G}$ for all $i$.

Under (i) and (ii) the decomposition (11) is unique up to the order of the idempotents $e_{h_{i}}$, and this was used by R. Exel to conclude that $\mathcal{S}(G)$ is an inverse semigroup [25, Theorem 3.4] (see also [26]).

The defining relations of $\mathcal{S}(G)$ are designed to guarantee that the map

$$
G \ni g \mapsto[g] \in \mathcal{S}(G)
$$

is a partial representation. More generally, we recall that a map $\pi: G \rightarrow S$, where $S$ is a monoid, is called a partial representation (or a partial homomorphism) if

(i) $\pi\left(1_{G}\right)=1_{S}$,

(ii) $\pi\left(g^{-1}\right) \pi(g) \pi(h)=\pi\left(g^{-1}\right) \pi(g h)$,

(iii) $\pi(g) \pi(h) \pi\left(h^{-1}\right)=\pi(g h) \pi\left(h^{-1}\right)$,

for all $g, h \in G$. There is an evident bijective correspondence between the partial homomorphisms from $G$ into a unital algebra $\mathcal{A}$ and the homomorphisms $K_{\mathrm{par}}(G) \rightarrow \mathcal{A}$.

The following fact, which is well known to the experts and whose verification is a direct exercise, results in a situation in which both partial cohomology theories are applicable.

Lemma 1.1. Let $(\mathcal{A}, \alpha)$ be a unital partial action of a group $G$ on an algebra $\mathcal{A}$. Then the map $\pi^{\alpha}: G \rightarrow \operatorname{End}_{K}(\mathcal{A}), g \mapsto \pi^{\alpha}$, given by

$$
\pi_{g}^{\alpha}(a)=\alpha_{g}\left(1_{g^{-1}} a\right)
$$

is a partial representation of $G$.

It follows that (2) induces a $K_{\text {par }}(G)$-module structure on $\mathcal{A}$ by means of

$$
[g] \cdot a=\pi_{g}^{\alpha}(a) .
$$




\section{The Alvares-Alves-Redondo cohomology}

2.1. The 0-th cohomology group. We begin with the situation of Lemma 1.1 with commutative $\mathcal{A}$, so that we shall consider partial $G$-modules in a more restricted sense than in [13. More precisely, by a partial $G$-module we shall mean a commutative partial $G$-module algebra, i.e. a pair $(\mathcal{A}, \alpha)$, where $\mathcal{A}$ is a commutative algebra over some fixed field $K$ and $\alpha$ is a partial action of $G$ on $\mathcal{A}$. Moreover, we shall assume that $\alpha$ is unital, which is also expressed by saying that the partial $G$-module is unital.

Recall from 13 that, given a unital partial $G$-module $(\mathcal{A}, \alpha)$, the 0 -th partial cohomology group $H^{0}(G, \mathcal{A})$ of $G$ with values in $(\mathcal{A}, \alpha)$ is

$$
H^{0}(G, \mathcal{A})=\left\{a \in \mathcal{U}(\mathcal{A}) \mid \forall g \in G: \alpha_{g}\left(1_{g^{-1}} a\right)=1_{g} a\right\}
$$

For a left $K_{\text {par }}(G)$-module $M$ the 0 -th cohomology group $H_{\text {par }}^{0}(G, M)$ of $G$ with values in $M$ was defined in [2] as

$$
H_{\text {par }}^{0}(G, M)=\left\{m \in M \mid \forall g \in G:[g] \cdot m=e_{g} \cdot m\right\},
$$

reminding that $e_{g}=[g]\left[g^{-1}\right] \in K_{\mathrm{par}}(G)$.

Proposition 2.1. Let $(\mathcal{A}, \alpha)$ be a unital partial $G$-module. Consider the induced $K_{\mathrm{par}}(G)$-module structure on the underlying $K$-vector space of $\mathcal{A}$. Then $H_{\text {par }}^{0}(G, \mathcal{A})$ is a unital $K$-subalgebra of $\mathcal{A}$ and

$$
H^{0}(G, \mathcal{A})=\mathcal{U}\left(H_{\text {par }}^{0}(G, \mathcal{A})\right) .
$$

Proof. Indeed, by (2), (3) and (5) an element $a \in \mathcal{A}$ belongs to $H_{\text {par }}^{0}(G, \mathcal{A})$ if and only if for every $g \in G$

$$
\alpha_{g}\left(1_{g^{-1}} a\right)=\alpha_{g}\left(1_{g^{-1}} \alpha_{g^{-1}}\left(1_{g} a\right)\right)
$$

the latter being $\alpha_{g}\left(\alpha_{g^{-1}}\left(1_{g} a\right)\right)=1_{g} a$. Comparing this with (4), we obtain the desired equality (6).

2.2. The 1-st cohomology group. Observe that the commutative subalgebra $B$ of $K_{\mathrm{par}}(G)$ considered in [2] (see also [8]) is exactly $K E(\mathcal{S}(G)$ ), where $E(\mathcal{S}(G)$ ) is the semilattice of idempotents of $\mathcal{S}(G)$. The action of $\mathcal{S}(G)$ on $E(\mathcal{S}(G))$ by conjugation

$$
s \cdot e=s e s^{-1}
$$

extends by linearity to a homomorphism $K_{\text {par }}(G) \rightarrow \operatorname{End}_{K}(B)$, yielding thus a $K_{\text {par }}(G)$-module structure on $B$. Let $\epsilon: K_{\text {par }}(G) \rightarrow B$ be the $K$-linear map defined on $s \in \mathcal{S}(G)$ by

$$
\epsilon(s)=s s^{-1} .
$$

Observe from (17) that $\epsilon$ is a morphism of $K_{\mathrm{par}}(G)$-modules, and it coincides with the one defined in [2] before Lemma 3.2.

We now recall an interpretation of $H_{p a r}^{1}(G, M)$ obtained in [2]. Given a $K_{\text {par }}(G)$ module $M$, a $K$-linear map $\delta: K_{\mathrm{par}}(G) \rightarrow M$ is called a partial derivation, if for all $s, t \in \mathcal{S}(G)$

$$
\delta(s t)=s \cdot \delta(t)+\epsilon(s t) \cdot \delta(s) .
$$

Notice that (9) is a corrected version of the definition given in [2].

\footnotetext{
${ }^{2}$ This is Hopf theoretic terminology (see [5]).
} 
The partial derivations of $K_{\mathrm{par}}(G)$ with values in $M$ form a $K$-space, which will be denoted by $\operatorname{Der}_{p a r}(G, M)$. A partial derivation $\delta \in \operatorname{Der}_{p a r}(G, M)$ is called principal (or inner), if there exists $m \in M$, such that for all $g \in G$

$$
\delta([g])=[g] \cdot m-e_{g} \cdot m .
$$

The $K$-subspace of the principal partial derivations will be denoted by $\operatorname{PDer}_{p a r}(G, M)$. Theorem 3.4 from [2] states that there is an isomorphism of additive groups

$$
H_{\text {par }}^{1}(G, M) \cong \operatorname{Der}_{\text {par }}(G, M) / \operatorname{PDer}_{\text {par }}(G, M) \text {. }
$$

We shall use the isomorphism (10) to give another interpretation of the elements of $H_{\text {par }}^{1}(G, M)$ in terms of certain maps $f: G \rightarrow M$ satisfying a kind of 1-cocycle identity.

Lemma 2.2. Let $\delta \in \operatorname{Der}_{p a r}(G, M)$. Then for any e $\in E(\mathcal{S}(G))$ we have

$$
\delta(e)=0 .
$$

Proof. Indeed, as $e^{2}=e$, we obtain by (8) and (9)

$$
\delta(e)=e \cdot \delta(e)+e \cdot \delta(e)=2 e \cdot \delta(e) .
$$

Multiplying the both sides of (12) by $e$, we obtain $e \cdot \delta(e)=2 e \cdot \delta(e)$, which gives $e \cdot \delta(e)=0$. Then (12) implies (11).

Lemma 2.3. For arbitrary $\delta \in \operatorname{Der}_{\text {par }}(G, M)$ and $s \in \mathcal{S}(G), e \in E(\mathcal{S}(G))$ one has

(i) $\delta(e s)=e \cdot \delta(s)$;

(ii) $\delta(s e)=s e s^{-1} \cdot \delta(s)$.

Proof. Indeed, applying (9) with $s=e$ and $t=s$ and then using (11) we obtain (i) Similarly the application of (9) with $t=e$ together with (11) gives (ii).

Lemma 2.4. Let $M$ be a $K_{\mathrm{par}}(G)$-module and $d: G \rightarrow M$ a map such that for all $g, h \in G$

$$
e_{g} \cdot d(g h)=[g] \cdot d(h)+e_{g h} \cdot d(g)
$$

Then the $K$-linear map $\delta: K_{\mathrm{par}}(G) \rightarrow M$ defined by

$$
\delta(e[g])=e \cdot d(g),
$$

where $e \in E(\mathcal{S}(G))$, is a partial derivation.

Proof. First of all, we show that $\delta$ is well defined. Applying (13) with $g=h=1_{G}$, we obtain $d\left(1_{G}\right)=0$. Then substituting $h=g^{-1}$ into (13), we have $d(g)=$ $-[g] \cdot d\left(g^{-1}\right)$, whence

$$
d(g)=e_{g} \cdot d(g)
$$

Now in view of (11) notice that $e[g]=f[h]$ in $\mathcal{S}(G)$ if and only if $g=h$ and $e_{g} e=e_{g} f$. In this case

$$
e \cdot d(g)=e e_{g} \cdot d(g)=f e_{g} \cdot d(g)=f \cdot d(g) .
$$

Consider two arbitrary elements $e[g]$ and $f[h]$ of $\mathcal{S}(G)$. Then their product is $e[g] f\left[g^{-1}\right][g h]$, so by (14)

$$
\delta(e[g] f[h])=e[g] f\left[g^{-1}\right] \cdot d(g h) .
$$

Now we calculate using (13)

$$
e[g] \cdot \delta(f[h])+\epsilon(e[g] f[h]) \cdot \delta(e[g])=e[g] f \cdot d(h)+\epsilon(e[g] f[h]) e \cdot d(g)
$$


GLOBALIZATION OF COHOMOLOGY IN THE SENSE OF ALVARES-ALVES-REDONDO 7

$$
\begin{aligned}
& =e[g] f\left[g^{-1}\right][g] \cdot d(h)+e[g] f\left[g^{-1}\right] e_{g h} e \cdot d(g) \\
& =e[g] f\left[g^{-1}\right] \cdot\left([g] \cdot d(h)+e_{g h} \cdot d(g)\right) \\
& =e[g] f\left[g^{-1}\right] \cdot\left(e_{g} \cdot d(g h)\right) \\
& =e[g] f\left[g^{-1}\right] \cdot d(g h),
\end{aligned}
$$

which in view of (16) shows that $\delta$ is a partial derivation.

Let us denote by $\mathrm{D}(G, M)$ the $K$-vector space of the maps $d: G \rightarrow M$ which satisfy (13).

Proposition 2.5. There is a bijective correspondence between the partial derivations of $K_{\mathrm{par}}(G)$ with values in $M$ and the elements of $\mathrm{D}(G, M)$.

Proof. Let $\delta \in \operatorname{Der}_{p a r}(G, M)$. By (8) and (9) and Lemma 2.3. (i) we obtain

$$
e_{g} \cdot \delta([g h])=\delta\left(e_{g}[g h]\right)=\delta([g][h])=[g] \cdot \delta([h])+e_{g h} \cdot \delta([g]) .
$$

So if we define $d: G \rightarrow M$ by

$$
d(g)=\delta([g]),
$$

then $d$ will satisfy (13). Conversely, if $d \in \mathrm{D}(G, M)$, then $\delta: K_{\text {par }}(G) \rightarrow M$ given by (14) is a partial derivation as was proved in Lemma 2.4 .

We now prove that the correspondence between $\delta$ and $d$ given by (14) and (17) is indeed bijective. If $d \mapsto \delta \mapsto d^{\prime}$, then

$$
d^{\prime}(g)=\delta([g])=\delta\left(1_{\mathcal{S}(G)}[g]\right)=1_{\mathcal{S}(G)} \cdot d(g)=d(g) .
$$

And if $\delta \mapsto d \mapsto \delta^{\prime}$, then

$$
\delta^{\prime}(e[g])=e \cdot d(g)=e \cdot \delta([g])=\delta(e[g]) .
$$

Let us introduce one more notation:

$$
\operatorname{PD}(G, M)=\left\{d: G \rightarrow M \mid \exists m \in M \forall g \in G: d(g)=[g] \cdot m-e_{g} \cdot m\right\} .
$$

Clearly, $\mathrm{PD}(G, M)$ is a $K$-subspace of $\mathrm{D}(G, M)$.

Theorem 2.6. Let $M$ be a $K_{\mathrm{par}}(G)$-module. Then $H_{\text {par }}^{1}(G, M)$ is isomorphic to the quotient of the additive group of $\mathrm{D}(G, M)$ modulo the subgroup $\operatorname{PD}(G, M)$.

Proof. This follows from (10) and Proposition 2.5 and the observation that the principal partial derivations of $K_{\mathrm{par}}(G)$ with values in $M$ correspond to the elements of $\operatorname{PD}(G, M)$.

Corollary 2.7. Let $(\mathcal{A}, \alpha)$ be a unital partial $G$-module. As in Proposition [2.1 we consider the corresponding $K_{\mathrm{par}}(G)$-module structure on $\mathcal{A}$. Then $H_{\text {par }}^{1}(G, \mathcal{A})$ is isomorphic to the quotient of the additive group of function $3^{3}$

$$
\left\{f: G \rightarrow \mathcal{A} \mid \forall g \in G: 1_{g} f(g h)=\alpha_{g}\left(1_{g^{-1}} f(h)\right)+1_{g h} f(g)\right\}
$$

by the subgroup

$$
\left\{f: G \rightarrow \mathcal{A} \mid \exists a \in \mathcal{A} \forall g \in G: f(g)=\alpha_{g}\left(1_{g^{-1}} a\right)-1_{g} a\right\} .
$$

\footnotetext{
${ }^{3}$ Observe that such functions automatically satisfy $f(g) \in 1_{g} \mathcal{A}$ in view of (15).
} 
Corollary 2.7permits us to compare $H_{\text {par }}^{1}(G, \mathcal{A})$ with the 1-st partial cohomology group $H^{1}(G, \mathcal{A})$. Recall from 13 that, in the setting of Corollary 2.7. $H^{1}(G, \mathcal{A})$ is the quotient of the multiplicative group of functions

$$
\left\{f: G \rightarrow \mathcal{A} \mid \forall g \in G: f(g) \in \mathcal{U}\left(1_{g} \mathcal{A}\right) \text { and } 1_{g} f(g h)=\alpha_{g}\left(1_{g^{-1}} f(h)\right) f(g)\right\}
$$

by the subgroup

$$
\left\{f: G \rightarrow \mathcal{A} \mid \exists a \in \mathcal{U}(\mathcal{A}) \forall g \in G: \quad f(g)=\alpha_{g}\left(1_{g^{-1}} a\right) a^{-1}\right\} .
$$

Thus, $H_{\text {par }}^{1}(G, \mathcal{A})$ is an "additive" analogue of $H^{1}(G, \mathcal{A})$.

2.3. A projective resolution of the $K_{\mathrm{par}}(G)$-module $B$. We are going to characterize the elements of $H_{\text {par }}^{n}(G, M)$ as classes of functions $f: G \rightarrow M$ satisfying certain $n$-cocycle identity, as we did in Subsection 2.2 for $n=1$. To this end, we adapt some ideas from [13] to the case of $K_{\mathrm{par}}(G)$-modules.

Lemma 2.8. Let $R$ be a unital ring and $\left\{e_{i}\right\}_{i \in I} \subseteq E(R)$ a set of idempotents of $R$. Then the left $R$-module $\bigoplus_{i \in I} R e_{i}$ is projective.

Proof. Indeed, each $R e_{i}$ is a projective left $R$-module, since $R e_{i} \oplus R\left(1_{R}-e_{i}\right)$ is isomorphic to $R$, a free $R$-module of rank 1 . Now, a direct sum of projective modules is projective (see, e.g., [29, Lemma 2.9 (iii)]).

Let $n \in \mathbb{N}$ and $g_{1}, \ldots, g_{n} \in G$. As in [13], we shall use the following notation:

$$
e_{\left(g_{1}, \ldots, g_{n}\right)}=e_{g_{1}} e_{g_{1} g_{2}} \ldots e_{g_{1} \ldots g_{n}} \in E(\mathcal{S}(G)) \text {. }
$$

Definition 2.9. Define

$$
\begin{aligned}
& P_{0}=K_{\mathrm{par}}(G), \\
& P_{n}=\bigoplus_{g_{1}, \ldots, g_{n} \in G} K_{\mathrm{par}}(G) e_{\left(g_{1}, \ldots, g_{n}\right)}, n \in \mathbb{N} .
\end{aligned}
$$

By Lemma 2.8 each $P_{n}$ is a projective $K_{\mathrm{par}}(G)$-module. It would be convenient to us to have an equivalent description of the modules $P_{n}$ which reminds the free resolution $R_{n}$ from [13].

Remark 2.10. For each $n \in \mathbb{N}$ the module $P_{n}$ is isomorphic, as a $K$-vector space, to the vector space over $K$ with basis

$$
\left\{s\left(g_{1}, \ldots, g_{n}\right) \mid s \in \mathcal{S}(G), g_{1}, \ldots, g_{n} \in G, s^{-1} s \leq e_{\left(g_{1}, \ldots, g_{n}\right)}\right\},
$$

where

$$
s\left(g_{1}, \ldots, g_{n}\right)=t\left(h_{1}, \ldots, h_{n}\right) \Leftrightarrow\left\{\begin{array}{l}
\left(g_{1}, \ldots, g_{n}\right)=\left(h_{1}, \ldots, h_{n}\right), \\
s e_{\left(g_{1}, \ldots, g_{n}\right)}=t e_{\left(h_{1}, \ldots, h_{n}\right)} .
\end{array}\right.
$$

Proof. Indeed, the elements $s e_{\left(g_{1}, \ldots, g_{n}\right)}$, where $s \in \mathcal{S}(G)$ and $g_{1}, \ldots, g_{n} \in G$, form a basis of the $K$-vector space $P_{n}$. Clearly, such $s e_{\left(g_{1}, \ldots, g_{n}\right)}$ may be identified with $s\left(g_{1}, \ldots, g_{n}\right)$, if (19) is assumed. It remains to observe that

$$
\exists s \in \mathcal{S}(G): t=s e_{\left(g_{1}, \ldots, g_{n}\right)} \Leftrightarrow t=t e_{\left(g_{1}, \ldots, g_{n}\right)} \Leftrightarrow t^{-1} t \leq e_{\left(g_{1}, \ldots, g_{n}\right)} .
$$

We extend the characterization of $P_{n}$ from Remark 2.10 to $n=0$ by identifying $P_{0}$ with the $K$-vector space with basis

$$
\{s() \mid s \in \mathcal{S}(G)\} .
$$


Definition 2.11. Define $K$-linear maps $\partial_{0}: P_{0} \rightarrow B$ and $\partial_{n}: P_{n} \rightarrow P_{n-1}, n \in \mathbb{N}$, as follows

$$
\begin{aligned}
\partial_{0}(s())= & s s^{-1} \\
\partial_{1}(s(g))= & s([g]()-()) \\
\partial_{n}\left(s\left(g_{1}, \ldots, g_{n}\right)\right)= & s\left(\left[g_{1}\right]\left(g_{2}, \ldots, g_{n}\right)\right. \\
& +\sum_{i=1}^{n-1}(-1)^{i}\left(g_{1}, \ldots, g_{i} g_{i+1}, \ldots, g_{n}\right) \\
& \left.+(-1)^{n}\left(g_{1}, \ldots, g_{n-1}\right)\right) .
\end{aligned}
$$

Observe that $\partial_{n}, n \in \mathbb{N} \cup\{0\}$, are morphisms of $K_{\text {par }}(G)$-modules. Indeed, this is trivial for $n \in \mathbb{N}$, and for $n=0$ one should remember that the $K_{\text {par }}(G)$-module structure on $B$ comes from the action of $\mathcal{S}(G)$ on $E(\mathcal{S}(G))$ by conjugation.

Our aim is to prove that Definition 2.11 gives a projective resolution of $B$ in the category of $K_{\text {par }}(G)$-modules. To this end, we shall show that the sequence $\left\{P_{n}\right\}_{n \geq-1}$, where $P_{-1}$ denotes $B$, admits a contracting homotopy similar to $\left\{\sigma_{n}\right\}_{n \geq-1}$ from [13. Definition 4.7]. Let $\eta: \mathcal{S}(G) \rightarrow G$ be the semigroup homomorphism which maps $e[g] \in \mathcal{S}(G)$ to $g$. As in [13, Lemma 4.8 (ii)], one can easily prove that

$$
s=s s^{-1}[\eta(s)] .
$$

Definition 2.12. Define $K$-linear maps $\sigma_{n}: P_{n} \rightarrow P_{n+1}, n \in \mathbb{N} \cup\{-1,0\}$, as follows

$$
\begin{aligned}
\sigma_{-1}(e) & =e(), \\
\sigma_{0}(s()) & =s s^{-1}(\eta(s)), \\
\sigma_{n}\left(s\left(g_{1}, \ldots, g_{n}\right)\right) & =s s^{-1}\left(\eta(s), g_{1}, \ldots, g_{n}\right), n \in \mathbb{N} .
\end{aligned}
$$

Since $s \leq[\eta(s)]$ by (24), we have that

$$
s s^{-1} \leq e_{\eta(s)}
$$

for all $s \in \mathcal{S}(G)$, and if moreover $s^{-1} s \leq e_{\left(g_{1}, \ldots, g_{n}\right)}$, then

$$
s s^{-1}=s\left(s^{-1} s\right) s^{-1} \leq[\eta(s)] s^{-1} s[\eta(s)]^{-1} \leq[\eta(s)] e_{\left(g_{1}, \ldots, g_{n}\right)}[\eta(s)]^{-1}=e_{\left(\eta(s), g_{1}, \ldots, g_{n}\right)} .
$$

Thus, $\sigma_{n}, n \in \mathbb{N} \cup\{-1,0\}$, are well defined.

Lemma 2.13. We have that

$$
\begin{aligned}
\partial_{0} \circ \sigma_{-1} & =\operatorname{id}_{B}, \\
\partial_{n+1} \circ \sigma_{n}+\sigma_{n-1} \circ \partial_{n} & =\operatorname{id}_{P_{n}}, n \in \mathbb{N} \cup\{0\} .
\end{aligned}
$$

Proof. It suffices to verify (27) and (28) on the $K$-basis (18) of $P_{n}, n \in \mathbb{N} \cup\{-1,0\}$. Equality (27) is a straightforward consequence of (21) and (25). To prove (28), one can follow the proof of [13, Lemma 4.9], remembering that $\Gamma(g)$ is now $[g]$ for all $g \in G$ and removing the unnecessary idempotents $\epsilon_{\left(g_{1}, \ldots, g_{n}\right)}$, where $g_{1}, \ldots, g_{n} \in$ G.

Proposition 2.14. The sequence

$$
\ldots \stackrel{\partial_{2}}{\rightarrow} P_{1} \stackrel{\partial_{1}}{\rightarrow} P_{0} \stackrel{\partial_{0}}{\rightarrow} B \rightarrow 0
$$

is a projective resolution of $B$ in the category of $K_{\mathrm{par}}(G)$-modules. 
Proof. In view of Lemma 2.8 we only need to prove that (29) is exact. Exactness in $B$ is just (27). The inclusion $\operatorname{ker} \partial_{n} \subseteq \operatorname{im} \partial_{n+1}, n \in \mathbb{N} \cup\{0\}$, is a trivial consequence of (28). For the converse inclusion, one may prove by induction on $n$ that $\partial_{n} \circ \partial_{n+1} \circ$ $\sigma_{n}=0$ (see, e.g., [28, p. 115]). This will guarantee that $\partial_{n} \circ \partial_{n+1}=0$, if we show that $\sigma_{n}\left(P_{n}\right)$ generates $P_{n+1}$ as a $K_{\text {par }}(G)$-module. Let $s\left(g_{1}, \ldots, g_{n+1}\right) \in P_{n+1}$. Consider $t=\left[\eta(s)^{-1}\right] s\left[g_{1}\right] \in \mathcal{S}(G)$. Since

$$
s e_{g_{1}}=s e_{\left(g_{1}, \ldots, g_{n+1}\right)} e_{g_{1}}=s e_{\left(g_{1}, \ldots, g_{n+1}\right)}=s
$$

by (20), we obtain using (24) that

$$
t t^{-1}=\left[\eta(s)^{-1}\right] s e_{g_{1}} s^{-1}[\eta(s)]=\left[\eta(s)^{-1}\right] s s^{-1}[\eta(s)]=s^{-1} s .
$$

Furthermore, by (26)

$$
e_{\eta(s)} s=e_{\eta(s)} s s^{-1} s=s s^{-1} s=s,
$$

whence

$$
t^{-1} t=\left[g_{1}^{-1}\right] s^{-1} e_{\eta(s)} s\left[g_{1}\right]=\left[g_{1}^{-1}\right] s^{-1} s\left[g_{1}\right] \leq\left[g_{1}^{-1}\right] e_{\left(g_{1}, \ldots, g_{n+1}\right)}\left[g_{1}\right] \leq e_{\left(g_{2}, \ldots, g_{n+1}\right)} .
$$

Finally,

$$
\eta(t)=\eta\left(\left[\eta(s)^{-1}\right] s\left[g_{1}\right]\right)=\eta(s)^{-1} \eta(s) g_{1}=g_{1} .
$$

Equalities (30) and (32) imply that

$$
s\left(g_{1}, \ldots, g_{n+1}\right)=s \cdot s^{-1} s\left(g_{1}, \ldots, g_{n+1}\right)=s \cdot \sigma_{n}\left(t\left(g_{2}, \ldots, g_{n+1}\right)\right)
$$

holds formally, and (31) is now used to show that $t\left(g_{2}, \ldots, g_{n+1}\right) \in P_{n}$, so that $\sigma_{n}\left(t\left(g_{2}, \ldots, g_{n+1}\right)\right)$ indeed makes sense.

Definition 2.15. Let $M$ be a $K_{\mathrm{par}}(G)$-module. Define the following additive groups

$$
\begin{aligned}
& C_{\text {par }}^{0}(G, M)=M, \\
& C_{\text {par }}^{n}(G, M)=\left\{f: G^{n} \rightarrow M \mid f\left(g_{1}, \ldots, g_{n}\right) \in e_{\left(g_{1}, \ldots, g_{n}\right)} \cdot M\right\}, n \in \mathbb{N} .
\end{aligned}
$$

Lemma 2.16. Let $M$ be a $K_{\mathrm{par}}(G)$-module. Then

$$
\operatorname{Hom}_{K_{\mathrm{par}}(G)}\left(P_{n}, M\right) \cong C_{\text {par }}^{n}(G, M) .
$$

Proof. The case $n=0$ is explained by the fact that $P_{0}$ is a free $K_{\mathrm{par}}(G)$-module of rank 1. Now let $n \in \mathbb{N}$ and observe using Definition 2.9 and the standard isomorphism $\operatorname{Hom}\left(\bigoplus A_{i},-\right) \cong \prod \operatorname{Hom}\left(A_{i},-\right)$ (see, for example, [28, p. 25]) that

$$
\begin{aligned}
\operatorname{Hom}_{K_{\mathrm{par}}(G)}\left(P_{n}, M\right) & \cong \prod_{g_{1}, \ldots, g_{n} \in G} \operatorname{Hom}_{K_{\mathrm{par}}(G)}\left(K_{\mathrm{par}}(G) e_{\left(g_{1}, \ldots, g_{n}\right)}, M\right) \\
& \cong \prod_{g_{g_{1}, \ldots, g_{n} \in G}} e_{\left(g_{1}, \ldots, g_{n}\right)} \cdot M \\
& \cong C_{p a r}^{n}(G, M) .
\end{aligned}
$$

Remark 2.17. With respect to the isomorphism from Lemma [2.16] any $\varphi \in$ $\operatorname{Hom}_{K_{\mathrm{par}}(G)}\left(P_{n}, M\right)$ is mapped to $f_{\varphi} \in C_{p a r}^{n}(G, M)$, where

$$
f_{\varphi}\left(g_{1}, \ldots, g_{n}\right)=\varphi\left(e_{\left(g_{1}, \ldots, g_{n}\right)}\left(g_{1}, \ldots, g_{n}\right)\right) .
$$


GLOBALIZATION OF COHOMOLOGY IN THE SENSE OF ALVARES-ALVES-REDONDO 11

Conversely, each $f^{\prime} \in C_{p a r}^{n}(G, M)$ corresponds to $\varphi \in \operatorname{Hom}_{K_{\mathrm{par}}(G)}\left(P_{n}, M\right)$ defined by

$$
\varphi\left(s\left(g_{1}, \ldots, g_{n}\right)\right)=s \cdot f^{\prime}\left(g_{1}, \ldots, g_{n}\right) .
$$

Definition 2.18. Let $M$ be a $K_{\mathrm{par}}(G)$-module and $n \in \mathbb{N} \cup\{0\}$. Define the $K$-linear map $\delta^{n}: C_{p a r}^{n}(G, M) \rightarrow C_{p a r}^{n+1}(G, M)$ as follows:

$$
\begin{aligned}
\left(\delta^{0} m\right)(g)= & {[g] \cdot m-e_{g} \cdot m, m \in C_{p a r}^{0}(G, M), } \\
\left(\delta^{n} f\right)\left(g_{1}, \ldots, g_{n+1}\right)= & {\left[g_{1}\right] \cdot f\left(g_{2}, \ldots, g_{n+1}\right) } \\
& +\sum_{i=1}^{n}(-1)^{i} e_{g_{1} \ldots g_{i}} \cdot f\left(g_{1}, \ldots, g_{i} g_{i+1}, \ldots, g_{n+1}\right) \\
& +(-1)^{n+1} e_{g_{1} \ldots g_{n+1}} \cdot f\left(g_{1}, \ldots, g_{n}\right), n \in \mathbb{N}, f \in C_{p a r}^{n}(G, M) .
\end{aligned}
$$

Lemma 2.19. For all $n \in \mathbb{N} \cup\{0\}$ and $f \in C_{\text {par }}^{n}(G, M)$ we have

$$
\delta^{n} f=f \circ \partial_{n+1},
$$

where $f$ and $\delta^{n} f$ are identified with the morphisms from $\operatorname{Hom}_{K_{\mathrm{par}}(G)}\left(P_{n}, M\right)$ as in Lemma 2.16. In particular,

$$
C_{\text {par }}^{0}(G, M) \stackrel{\delta^{0}}{\rightarrow} C_{\text {par }}^{1}(G, M) \stackrel{\delta^{1}}{\rightarrow} \ldots
$$

is a cochain complex of abelian groups.

Proof. It suffices to verify (37) on the generators

$$
\left\{e_{\left(g_{1}, \ldots, g_{n+1}\right)}\left(g_{1}, \ldots, g_{n+1}\right) \mid g_{1}, \ldots, g_{n+1} \in G\right\}
$$

of $P_{n+1}$. We first consider the case $n=0$. Let $m \in C_{\text {par }}^{0}(G, M)$. Then, as an element of $\operatorname{Hom}_{K_{\mathrm{par}}(G)}\left(P_{0}, M\right), m$ sends $s()$ to $s \cdot m$. Using (22), (34) and (35), we have

$$
\begin{aligned}
m \circ \partial_{1}\left(e_{g}(g)\right) & =m\left(e_{g}([g]()-())\right)=m\left([g]()-e_{g}()\right) \\
& =[g] \cdot m-e_{g} \cdot m=\left(\delta^{0} m\right)(g) \\
& =e_{g} \cdot\left(\delta^{0} m\right)(g)=\left(\delta^{0} m\right)\left(e_{g}(g)\right) .
\end{aligned}
$$

Now let $n \in \mathbb{N}$ and $f$ be a function from $C_{\text {par }}^{n}(G, M)$. By (23), (33), (34) and (36)

$$
\begin{aligned}
f \circ \partial_{n+1}\left(e_{\left(g_{1}, \ldots, g_{n+1}\right)}\left(g_{1}, \ldots, g_{n+1}\right)\right)= & e_{\left(g_{1}, \ldots, g_{n+1}\right)} \cdot\left(\left[g_{1}\right] \cdot f\left(g_{2}, \ldots, g_{n+1}\right)\right. \\
& +\sum_{i=1}^{n}(-1)^{i} f\left(g_{1}, \ldots, g_{i} g_{i+1}, \ldots, g_{n+1}\right) \\
& \left.+(-1)^{n+1} f\left(g_{1}, \ldots, g_{n}\right)\right) \\
= & e_{\left(g_{1}, \ldots, g_{n+1}\right)} \cdot\left(\left[g_{1}\right] \cdot f\left(g_{2}, \ldots, g_{n+1}\right)\right. \\
& +\sum_{i=1}^{n}(-1)^{i} e_{g_{1} \ldots g_{i}} \cdot f\left(g_{1}, \ldots, g_{i} g_{i+1}, \ldots, g_{n+1}\right) \\
& \left.+(-1)^{n+1} e_{g_{1} \ldots g_{n+1}} \cdot f\left(g_{1}, \ldots, g_{n}\right)\right) \\
= & \left(\delta^{n} f\right)\left(e_{\left(g_{1}, \ldots, g_{n+1}\right)}\left(g_{1}, \ldots, g_{n+1}\right)\right) .
\end{aligned}
$$


Definition 2.20. Denote $\operatorname{ker} \delta^{n}$ by $Z_{\text {par }}^{n}(G, M), n \in \mathbb{N} \cup\{0\}$, and $\operatorname{im} \delta^{n}$ by $B_{\text {par }}^{n}(G, M), n \in \mathbb{N}$, where $\delta^{n}$ is given by (35) and (36).

Theorem 2.21. Let $G$ be a group and $M$ a $K_{\text {par }}(G)$-module. Then $H_{\text {par }}^{0}(G, M) \cong$ $Z_{\text {par }}^{0}(G, M)$ and $H_{\text {par }}^{n}(G, M) \cong Z_{\text {par }}^{n}(G, M) / B_{\text {par }}^{n}(G, M)$.

Proof. This follows from Proposition 2.14 and Lemmas 2.16 and 2.19.

Remark 2.22. For a $K_{\mathrm{par}}(G)$-module $\mathcal{A}$ coming from a partial $G$-module $(\mathcal{A}, \alpha)$ and $n \in \mathbb{N}$ we have in view of Lemma 1.1

$$
C_{p a r}^{n}(G, \mathcal{A})=\left\{f: G^{n} \rightarrow \mathcal{A} \mid f\left(g_{1}, \ldots, g_{n}\right) \in 1_{\left(g_{1}, \ldots, g_{n}\right)} \mathcal{A}\right\},
$$

where

$$
1_{\left(g_{1}, \ldots, g_{n}\right)}=1_{g_{1}} 1_{g_{1} g_{2}} \ldots 1_{g_{1} \ldots g_{n}} \in \mathcal{A} \text {. }
$$

Then formulas (35) and (36) take the following form

$$
\begin{aligned}
\left(\delta^{0} a\right)(g)= & \alpha_{g}\left(1_{g^{-1}} a\right)-1_{g} a, a \in C_{p a r}^{0}(G, \mathcal{A})=\mathcal{A}, \\
\left(\delta^{n} f\right)\left(g_{1}, \ldots, g_{n+1}\right)= & \alpha_{g_{1}}\left(1_{g_{1}^{-1}} f\left(g_{2}, \ldots, g_{n+1}\right)\right) \\
& +\sum_{i=1}^{n}(-1)^{i} 1_{g_{1} \ldots g_{i}} f\left(g_{1}, \ldots, g_{i} g_{i+1}, \ldots, g_{n+1}\right) \\
& +(-1)^{n+1} 1_{g_{1} \ldots g_{n+1}} f\left(g_{1}, \ldots, g_{n}\right),
\end{aligned}
$$

$n \in \mathbb{N}, f \in C_{p a r}^{n}(G, M)$.

\section{Globalization}

3.1. From globalization to an extendibility property. Throughout this section $\alpha$ will be a unital partial action of a group $G$ on a (unital) algebra $\mathcal{A}$. We regard $\mathcal{A}$ as a $K_{\text {par }}(G)$-module in a natural way (see Lemma 1.1). We also fix $(\mathcal{B}, \beta)$ an enveloping action of $\alpha$ (see [8, Definition 4.2]) with an injective morphism $\varphi:(\mathcal{A}, \alpha) \rightarrow(\mathcal{B}, \beta)$. The algebra $\mathcal{B}$ does not always have an identity element, and for our technique we need to have a unital algebra. Instead of assuming that $\mathcal{B}$ has $1_{\mathcal{B}}$, we shall work more generally with the multiplier algebra $\mathcal{M}(\mathcal{B})$ of $\mathcal{B}$.

We recall that the multiplier algebra $\mathcal{M}(\mathcal{B})$ of an algebra $\mathcal{B}$ is the set

$$
\mathcal{M}(\mathcal{B})=\left\{(R, L) \in \operatorname{End}\left({ }_{\mathcal{B}} \mathcal{B}\right) \times \operatorname{End}\left(\mathcal{B}_{\mathcal{B}}\right):(a R) b=a(L b) \text { for all } a, b \in \mathcal{B}\right\}
$$

with component-wise addition and multiplication (for more details see [7, 8). Here we use the right-hand side notation for left $\mathcal{B}$-module homomorphisms, i.e. we write $b \mapsto b \gamma$ for $\gamma:{ }_{\mathcal{B}} \mathcal{B} \rightarrow{ }_{\mathcal{B}} \mathcal{B}$, while for a right $\mathcal{B}$-module homomorphism $\gamma: \mathcal{B}_{\mathcal{B}} \rightarrow \mathcal{B}_{\mathcal{B}}$ the usual notation is used: $b \mapsto \gamma b$.

For a multiplier $\gamma=(R, L) \in \mathcal{M}(\mathcal{B})$ and $b \in \mathcal{B}$ we set $b \gamma=b R$ and $\gamma b=L b$. Thus one always has $(a \gamma) b=a(\gamma b)$ for arbitrary $a, b \in \mathcal{B}$.

The action $\beta$ induces an action $\beta^{*}$ of $G$ on $\mathcal{M}(\mathcal{B})$, where $\beta_{g}^{*}(u)=\beta_{g} u \beta_{g}^{-1}$ for $u \in \mathcal{M}(\mathcal{B})$ and $g \in G$. Denote by $C^{n}(G, \mathcal{M}(\mathcal{B})), Z^{n}(G, \mathcal{M}(\mathcal{B})), B^{n}(G, \mathcal{M}(\mathcal{B}))$ and $H^{n}(G, \mathcal{M}(\mathcal{B}))$ the corresponding (abelian) groups of $n$-cochains, $n$-cocycles, $n$ coboundaries and $n$-cohomologies of $G$ with values in the additive group of $\mathcal{M}(\mathcal{B})$.

Definition 3.1. Given $n \in \mathbb{N}$ and $u \in C^{n}(G, \mathcal{M}(\mathcal{B}))$, define the restriction of $u$ to $\mathcal{A}$ to be the map $w: G^{n} \rightarrow \mathcal{A}$, such that

$$
\varphi\left(w\left(g_{1}, \ldots, g_{n}\right)\right)=\varphi\left(1_{\left(g_{1}, \ldots, g_{n}\right)}\right) u\left(g_{1}, \ldots, g_{n}\right),
$$


where $g_{1}, \ldots, g_{n} \in G$. If $n=0$ and $u \in C^{0}(G, \mathcal{M}(\mathcal{B}))=\mathcal{M}(\mathcal{B})$, then $w$ is the element of $\mathcal{A}$, satisfying (41), in which $1_{\left(g_{1}, \ldots, g_{n}\right)}$ means $1_{\mathcal{A}}$.

Notice that in (41) we could replace $\varphi\left(1_{\left(g_{1}, \ldots, g_{n}\right)}\right) u\left(g_{1}, \ldots, g_{n}\right)$ by its "left version" $u\left(g_{1}, \ldots, g_{n}\right) \varphi\left(1_{\left(g_{1}, \ldots, g_{n}\right)}\right)$. But in fact the two versions coincide. Indeed, $\varphi(\mathcal{A})$ is an ideal of $\mathcal{B}$, so $u$ restricted to $\varphi(\mathcal{A})$ is a multiplier of $\varphi(\mathcal{A})$. Since $\varphi\left(1_{\left(g_{1}, \ldots, g_{n}\right)}\right)$ is a central idempotent of $\varphi(\mathcal{A})$, we have $\varphi\left(1_{\left(g_{1}, \ldots, g_{n}\right)}\right) u\left(g_{1}, \ldots, g_{n}\right)=$ $\varphi\left(u\left(g_{1}, \ldots, g_{n}\right) 1_{\left(g_{1}, \ldots, g_{n}\right)}\right)$ by [14, Remark 5.2]. The observation that the multipliers of $\mathcal{B}$ (and of $\varphi(\mathcal{A})$ as well) "commute" with central idempotents of $\varphi(\mathcal{A})$ will be implicitly used several times in what follows.

We will write $\rho(u)=w$ when $w$ is a restriction of $u$. Clearly, $\rho(u) \in C_{\text {par }}^{n}(G, \mathcal{A})$, as the right-hand side of (41) is stable under the multiplication by $\varphi\left(1_{\left(g_{1}, \ldots, g_{n}\right)}\right)$ on the left.

Proposition 3.2. The restriction map $\rho: C^{n}(G, \mathcal{M}(\mathcal{B})) \rightarrow C_{\text {par }}^{n}(G, \mathcal{A})$ induces a homomorphism of the cohomology groups $H^{n}(G, \mathcal{M}(\mathcal{B})) \rightarrow H_{\text {par }}^{n}(G, \mathcal{A})$.

Proof. It is readily seen by (41) that $\rho$ is a homomorphism, so we only need to show that $\rho$ commutes with the coboundary operators. Let $n=0$ and $u \in C^{0}(G, \mathcal{M}(\mathcal{B}))=\mathcal{M}(\mathcal{B})$. Then for all $g \in G$ by (41) and the fact that $\varphi$ is a morphism of partial actions we have

$$
\begin{aligned}
\varphi\left(\left(\delta^{0} \rho(u)\right)(g)\right) & =\varphi\left(\alpha_{g}\left(1_{g^{-1}} \rho(u)\right)-1_{g} \rho(u)\right) \\
& =\beta_{g}\left(\varphi\left(1_{g^{-1}}\right) \varphi(\rho(u))\right)-\varphi\left(1_{g}\right) \varphi(\rho(u)) \\
& =\beta_{g}\left(\varphi\left(1_{g^{-1}}\right) \varphi\left(1_{\mathcal{A}}\right) u\right)-\varphi\left(1_{g}\right) \varphi\left(1_{\mathcal{A}}\right) u \\
& =\varphi\left(1_{g}\right)\left(\beta_{g}^{*}(u)-u\right) \\
& =\varphi\left(1_{g}\right)\left(\delta^{0} u\right)(g) \\
& =\varphi\left(\rho\left(\delta^{0} u\right)(g)\right),
\end{aligned}
$$

whence $\delta^{0} \rho(u)=\rho\left(\delta^{0} u\right)$.

Consider now $n \in \mathbb{N}$ and $u \in C^{n}(G, \mathcal{M}(\mathcal{B}))$. For arbitrary $g_{1}, \ldots, g_{n+1} \in G$, using (41) as above, one has

$$
\begin{aligned}
\varphi\left(\left(\delta^{n} \rho(u)\right)\left(g_{1}, \ldots, g_{n+1}\right)\right)= & \varphi\left(\alpha_{g_{1}}\left(1_{g_{1}^{-1}} \rho(u)\left(g_{2}, \ldots, g_{n+1}\right)\right)\right. \\
& +\sum_{i=1}^{n}(-1)^{i} 1_{g_{1} \ldots g_{i}} \rho(u)\left(g_{1}, \ldots, g_{i} g_{i+1}, \ldots, g_{n+1}\right) \\
& \left.+(-1)^{n+1} 1_{g_{1} \ldots g_{n+1}} \rho(u)\left(g_{1}, \ldots, g_{n}\right)\right) \\
= & \beta_{g_{1}}\left(\varphi\left(1_{g_{1}^{-1}}\right) \varphi\left(\rho(u)\left(g_{2}, \ldots, g_{n+1}\right)\right)\right) \\
& +\sum_{i=1}^{n}(-1)^{i} \varphi\left(1_{g_{1} \ldots g_{i}}\right) \varphi\left(\rho(u)\left(g_{1}, \ldots, g_{i} g_{i+1}, \ldots, g_{n+1}\right)\right) \\
& +(-1)^{n+1} \varphi\left(1_{g_{1} \ldots g_{n+1}}\right) \varphi\left(\rho(u)\left(g_{1}, \ldots, g_{n}\right)\right) \\
= & \beta_{g_{1}}\left(\varphi\left(1_{g_{1}^{-1}}\right) \varphi\left(1_{\left(g_{2}, \ldots, g_{n+1}\right)}\right) u\left(g_{2}, \ldots, g_{n+1}\right)\right) \\
& +\sum_{i=1}^{n}(-1)^{i} \varphi\left(1_{g_{1} \ldots g_{i}}\right) \varphi\left(1_{\left(g_{1}, \ldots, g_{i} g_{i+1}, \ldots, g_{n+1}\right)}\right) u\left(g_{1}, \ldots, g_{i} g_{i+1}, \ldots, g_{n+1}\right)
\end{aligned}
$$




$$
\begin{aligned}
& +(-1)^{n+1} \varphi\left(1_{g_{1} \ldots g_{n+1}}\right) \varphi\left(1_{\left(g_{1}, \ldots, g_{n}\right)}\right) u\left(g_{1}, \ldots, g_{n}\right) \\
= & 1_{\left(g_{1}, \ldots, g_{n+1}\right)}\left(\beta_{g_{1}}^{*}\left(u\left(g_{2}, \ldots, g_{n+1}\right)\right)\right. \\
& +\sum_{i=1}^{n}(-1)^{i} u\left(g_{1}, \ldots, g_{i} g_{i+1}, \ldots, g_{n+1}\right) \\
& \left.+(-1)^{n+1} u\left(g_{1}, \ldots, g_{n}\right)\right) \\
= & 1_{\left(g_{1}, \ldots, g_{n+1}\right)}\left(\delta^{n} u\right)\left(g_{1}, \ldots, g_{n+1}\right) \\
= & \varphi\left(\rho\left(\delta^{n} u\right)\left(g_{1}, \ldots, g_{n+1}\right)\right),
\end{aligned}
$$

so that $\delta^{n} \rho(u)=\rho\left(\delta^{n} u\right)$.

Definition 3.3. Given $w \in Z_{\text {par }}^{n}(G, \mathcal{A})$, by a globalization of $w$ we mean $u \in$ $Z^{n}(G, \mathcal{M}(\mathcal{B}))$ satisfying (41). If $w$ admits a globalization, then we say that $w$ is globalizable.

Recall that the enveloping action $(\mathcal{B}, \beta)$ for $(\mathcal{A}, \alpha)$ was constructed in $[8]$ as the restriction of the global action $(\mathcal{F}, \beta)$ to the subalgebra

$$
\mathcal{B}=\sum_{g \in G} \beta_{g}(\varphi(\mathcal{A}))
$$

Here $\mathcal{F}$ is the ring of functions $G \rightarrow \mathcal{A}$ and

$$
\left.\beta_{g}(f)\right|_{t}=f\left(g^{-1} t\right)
$$

for all $x, t \in G$, where the notation $\left.f\right|_{t}$ from $[8$ is used for the value $f(t)$ of $f \in \mathcal{F}$ at $t \in G$. The injective morphism $\varphi: \mathcal{A} \rightarrow \mathcal{F}$ is then defined by the formula

$$
\left.\varphi(a)\right|_{t}=\alpha_{t^{-1}}\left(1_{t} a\right) .
$$

Clearly, $\varphi(\mathcal{A}) \subseteq \mathcal{B}$, so $\varphi$ is a morphism $(\mathcal{A}, \alpha) \rightarrow(\mathcal{B}, \beta)$ too. Since all enveloping actions of $(\mathcal{A}, \alpha)$ are isomorphic $[8]$ to each other, we may always assume that $(\mathcal{B}, \beta)$ and $\varphi$ are of this form.

Lemma 3.4. Any $w \in Z_{\text {par }}^{0}(G, \mathcal{A})$ is uniquely globalizable.

Proof. Define $u \in C^{0}(G, \mathcal{F})=\mathcal{F}$ to be the constant function taking the value $w \in \mathcal{A}$ at any $t \in G$. Using (39) and (44), we obtain

$$
\left.\left.\varphi\left(1_{\mathcal{A}}\right)\right|_{t} u\right|_{t}=1_{t^{-1}} w=\alpha_{t^{-1}}\left(1_{t} w\right)=\left.\varphi(w)\right|_{t},
$$

yielding (41). The proof of the formula $\left.\left.\beta_{g}(\varphi(a))\right|_{t} u\right|_{t}=\left.\beta_{g}(\varphi(a w))\right|_{t}$ from [16, Remark 2.3] works here without any change, so $\beta_{g}(\varphi(\mathcal{A})) u \subseteq \varphi(\mathcal{A})$. Hence $\mathcal{B} u \subseteq \mathcal{B}$ by (42). In a similar way $\left.\left.u\right|_{t} \beta_{g}(\varphi(a))\right|_{t}=\left.\beta_{g}(\varphi(w a))\right|_{t}$, which implies $u \mathcal{B} \subseteq \mathcal{B}$, and thus $u \in C^{0}(G, \mathcal{M}(\mathcal{B}))$. To prove the 0 -cocycle identity $\beta_{g}^{*}(u)=u$ for $u$, it suffices to show that $\beta_{g}(u f)=u \beta_{g}(f)$ for any $f \in \mathcal{F}$. We have by (43)

$$
\left.\beta_{g}(u f)\right|_{t}=\left.(u f)\right|_{g^{-1} t}=\left.\left.u\right|_{g^{-1} t} f\right|_{g^{-1} t}=\left.\left.u\right|_{t} \beta_{g}(f)\right|_{t},
$$

whence $u \in Z^{0}(G, \mathcal{M}(\mathcal{B}))$.

The uniqueness of $u$ is proved the same way as in [16, Remark 2.3].

For the case $w \in Z_{p a r}^{n}(G, \mathcal{A}), n \in \mathbb{N}$, we shall need an "additive" version of [16, Lemma 2.1]. 
GLOBALIZATION OF COHOMOLOGY IN THE SENSE OF ALVARES-ALVES-REDONDO 15

Lemma 3.5. Let $\widetilde{w} \in C^{n}(G, \mathcal{A})$. Then $u \in C^{n}(G, \mathcal{F})$, defined by

$$
\begin{aligned}
\left.u\left(g_{1}, \ldots, g_{n}\right)\right|_{t}= & (-1)^{n} \widetilde{w}\left(t^{-1}, g_{1}, \ldots, g_{n-1}\right)+\widetilde{w}\left(t^{-1} g_{1}, g_{2}, \ldots, g_{n}\right) \\
& +\sum_{i=1}^{n-1}(-1)^{i} \widetilde{w}\left(t^{-1}, g_{1}, \ldots, g_{i} g_{i+1}, \ldots, g_{n}\right),
\end{aligned}
$$

is an $n$-cocycle with respect to the action $\beta$ of $G$ on $\mathcal{F}$.

Proof. Observe by (45) that

$$
\left.u\left(g_{1}, \ldots, g_{n}\right)\right|_{t}=\widetilde{w}\left(g_{1}, \ldots, g_{n}\right)-\left(\tilde{\delta}^{n} \widetilde{w}\right)\left(t^{-1}, g_{1}, \ldots, g_{n}\right),
$$

where $\tilde{\delta}^{n}: C^{n}(G, \mathcal{A}) \rightarrow C^{n+1}(G, \mathcal{A})$ is the coboundary operator which corresponds to the trivial $G$-module, i.e.

$$
\begin{aligned}
\left(\tilde{\delta}^{n} \widetilde{w}\right)\left(g_{1}, \ldots, g_{n+1}\right)= & \widetilde{w}\left(g_{2}, \ldots, g_{n+1}\right) \\
& +\sum_{i=1}^{n}(-1)^{i} \widetilde{w}\left(g_{1}, \ldots, g_{i} g_{i+1}, \ldots, g_{n+1}\right) \\
& +(-1)^{n+1} \widetilde{w}\left(g_{1}, \ldots, g_{n}\right) .
\end{aligned}
$$

Calculating the value of $\left(\delta^{n} u\right)\left(g_{1}, \ldots, g_{n+1}\right)$ at $t \in G$, we obtain using (43)

$$
\begin{aligned}
\left.u\left(g_{2}, \ldots, g_{n+1}\right)\right|_{g_{1}^{-1} t} & +\left.\sum_{i=1}^{n}(-1)^{i} u\left(g_{1}, \ldots, g_{i} g_{i+1}, \ldots, g_{n+1}\right)\right|_{t} \\
& +\left.(-1)^{n+1} u\left(g_{1}, \ldots, g_{n}\right)\right|_{t}
\end{aligned}
$$

which in view of (46) equals

$$
\begin{aligned}
& \widetilde{w}\left(g_{2}, \ldots, g_{n+1}\right)-\left(\tilde{\delta}^{n} \widetilde{w}\right)\left(t^{-1} g_{1}, g_{2}, \ldots, g_{n+1}\right) \\
& +\sum_{i=1}^{n}(-1)^{i} \widetilde{w}\left(g_{1}, \ldots, g_{i} g_{i+1}, \ldots, g_{n+1}\right) \\
& +\sum_{i=1}^{n}(-1)^{i+1}\left(\tilde{\delta}^{n} \widetilde{w}\right)\left(t^{-1}, g_{1}, \ldots, g_{i} g_{i+1}, \ldots, g_{n+1}\right) \\
& +(-1)^{n+1} \widetilde{w}\left(g_{1}, \ldots, g_{n}\right)+(-1)^{n}\left(\tilde{\delta}^{n} \widetilde{w}\right)\left(t^{-1}, g_{1}, \ldots, g_{n}\right) .
\end{aligned}
$$

The latter is readily seen to be $\left(\tilde{\delta}^{n+1} \tilde{\delta}^{n} \widetilde{w}\right)\left(t^{-1}, g_{1}, \ldots, g_{n+1}\right)=0_{\mathcal{A}}$.

As in [16, Theorem 2.4], the existence of a globalization of $w \in Z_{p a r}^{n}(G, \mathcal{A})$ is equivalent to certain extendibility property. For any $f \in C^{n}(G, \mathcal{A})$ define

$$
\begin{aligned}
\left(\tilde{\delta}^{n} f\right)\left(g_{1}, \ldots, g_{n+1}\right)= & \alpha_{g_{1}}\left(1_{g_{1}^{-1}} f\left(g_{2}, \ldots, g_{n+1}\right)\right) \\
& +\sum_{i=1}^{n}(-1)^{i} 1_{g_{1}} f\left(g_{1}, \ldots, g_{i} g_{i+1}, \ldots, g_{n+1}\right) \\
& +(-1)^{n+1} 1_{g_{1}} f\left(g_{1}, \ldots, g_{n}\right) .
\end{aligned}
$$

Theorem 3.6. A cocycle $w \in Z_{\text {par }}^{n}(G, \mathcal{A}), n \in \mathbb{N}$, is globalizable if and only if there exists $\widetilde{w} \in C^{n}(G, \mathcal{A})$ such that

$$
\tilde{\delta}^{n} \widetilde{w}=0
$$


and

$$
w\left(g_{1}, \ldots, g_{n}\right)=1_{\left(g_{1}, \ldots, g_{n}\right)} \widetilde{w}\left(g_{1}, \ldots, g_{n}\right),
$$

for all $g_{1}, \ldots, g_{n} \in G$.

Proof. If $w$ is globalizable and $u \in Z^{n}(G, \mathcal{M}(\mathcal{B}))$ is its globalization, then as in the proof of [16, Theorem 2.4] we define

$$
\varphi\left(\widetilde{w}\left(g_{1}, \ldots, g_{n}\right)\right)=\varphi\left(1_{\mathcal{A}}\right) u\left(g_{1}, \ldots, g_{n}\right)=u\left(g_{1}, \ldots, g_{n}\right) \varphi\left(1_{\mathcal{A}}\right) .
$$

Clearly, $w\left(g_{1}, \ldots, g_{n}\right) \in \mathcal{A}$, since $\varphi(\mathcal{A})$ is an ideal in $\mathcal{B}$, and moreover (49) is satisfied. Using the formula

$$
\beta_{g_{1}}^{*}\left(u\left(g_{2}, \ldots, g_{n+1}\right)\right) \varphi\left(1_{g_{1}}\right)=\varphi\left(\alpha_{g_{1}}\left(1_{g_{1}^{-1}} \widetilde{w}\left(g_{2}, \ldots, g_{n+1}\right)\right)\right),
$$

which follows from (49) as in the proof of [16, Theorem 2.4], we obtain (48) by applying both sides of the cocycle identity

$$
\begin{aligned}
\beta_{g_{1}}^{*}\left(u\left(g_{2}, \ldots, g_{n+1}\right)\right) & +\sum_{i=1}^{n}(-1)^{i} u\left(g_{1}, \ldots, g_{i} g_{i+1}, \ldots, g_{n+1}\right) \\
& +(-1)^{n+1} u\left(g_{1}, \ldots, g_{n}\right)=0
\end{aligned}
$$

to $\varphi\left(1_{g_{1}}\right)$.

Conversely, given $\widetilde{w} \in C^{n}(G, \mathcal{A})$ satisfying (48) and (49), define $u \in C^{n}(G, \mathcal{F})$ by (45). We immediately have $u \in Z^{n}(G, \mathcal{F})$ by Lemma 3.5. Now, using (44), (45) and (49) and the cocycle identity for $w$, we obtain

$$
\begin{aligned}
\left.\varphi\left(w\left(g_{1}, \ldots, g_{n}\right)\right)\right|_{t}= & \alpha_{t^{-1}}\left(1_{t} w\left(g_{1}, \ldots, g_{n}\right)\right) \\
= & 1_{t^{-1}} w\left(t^{-1} g_{1}, g_{2}, \ldots, g_{n}\right) \\
& +\sum_{i=1}^{n-1}(-1)^{i} 1_{t^{-1} g_{1} \ldots g_{i}} w\left(t^{-1}, g_{1}, \ldots, g_{i} g_{i+1}, \ldots, g_{n}\right) \\
& +(-1)^{n} 1_{t^{-1} g_{1} \ldots g_{n}} w\left(t^{-1}, g_{1}, \ldots, g_{n-1}\right) \\
= & 1_{t^{-1}} 1_{\left(t^{-1} g_{1}, g_{2}, \ldots, g_{n}\right)} \widetilde{w}\left(t^{-1} g_{1}, g_{2}, \ldots, g_{n}\right) \\
& +\sum_{i=1}^{n-1}(-1)^{i} 1_{t^{-1} g_{1} \ldots g_{i}} 1_{\left(t^{-1}, g_{1}, \ldots, g_{i} g_{i+1}, \ldots, g_{n}\right)} \widetilde{w}\left(t^{-1}, g_{1}, \ldots, g_{i} g_{i+1}, \ldots, g_{n}\right) \\
& +(-1)^{n} 1_{t^{-1}} g_{1} \ldots g_{n} 1_{\left(t^{-1}, g_{1}, \ldots, g_{n-1}\right)} \widetilde{w}\left(t^{-1}, g_{1}, \ldots, g_{n-1}\right) \\
= & \left.1_{\left(t^{-1}, g_{1}, \ldots, g_{n}\right)} u\left(g_{1}, \ldots, g_{n}\right)\right|_{t} \\
= & \left.\left.\varphi\left(1_{\left(g_{1}, \ldots, g_{n}\right)}\right)\right|_{t} u\left(g_{1}, \ldots, g_{n}\right)\right|_{t},
\end{aligned}
$$

whence (41).

We have yet to prove that $u\left(g_{1}, \ldots, g_{n}\right) \in \mathcal{M}(\mathcal{B})$, i.e.

$$
u\left(g_{1}, \ldots, g_{n}\right) \mathcal{B}, \mathcal{B} u\left(g_{1}, \ldots, g_{n}\right) \subseteq \mathcal{B}
$$

for all $g_{1}, \ldots, g_{n} \in G$. Since by (45) and (48)

$$
\begin{aligned}
\left.1_{t^{-1}} u\left(g_{1}, \ldots, g_{n}\right)\right|_{t}= & 1_{t^{-1}} \widetilde{w}\left(t^{-1} g_{1}, g_{2}, \ldots, g_{n}\right) \\
& +\sum_{i=1}^{n-1}(-1)^{i} 1_{t^{-1}} \widetilde{w}\left(t^{-1}, g_{1}, \ldots, g_{i} g_{i+1}, \ldots, g_{n}\right) \\
& +(-1)^{n} 1_{t^{-1}} \widetilde{w}\left(t^{-1}, g_{1}, \ldots, g_{n-1}\right)
\end{aligned}
$$




$$
=\alpha_{t^{-1}}\left(1_{t} \widetilde{w}\left(g_{1}, \ldots, g_{n}\right)\right)
$$

it follows that

$$
\left.\left.u\left(g_{1}, \ldots, g_{n}\right)\right|_{t} \varphi(a)\right|_{t}=\alpha_{t^{-1}}\left(1_{t} \widetilde{w}\left(g_{1}, \ldots, g_{n}\right)\right) \alpha_{t^{-1}}\left(1_{t} a\right)=\left.\varphi\left(\widetilde{w}\left(g_{1}, \ldots, g_{n}\right) a\right)\right|_{t},
$$

whence

$$
u\left(g_{1}, \ldots, g_{n}\right) \varphi(\mathcal{A}) \subseteq \varphi(\mathcal{A})
$$

Now $u$, being an $n$-cocycle with values in $(\mathcal{F}, \beta)$, satisfies

$$
\begin{aligned}
\beta_{t^{-1}}\left(u\left(g_{1}, \ldots, g_{n}\right)\right) \varphi(a)= & u\left(t^{-1} g_{1}, g_{2}, \ldots, g_{n}\right) \varphi(a) \\
& +\sum_{i=1}^{n-1}(-1)^{i} u\left(t^{-1}, g_{1}, \ldots, g_{i} g_{i+1}, \ldots, g_{n+1}\right) \varphi(a) \\
& +(-1)^{n} u\left(t^{-1}, g_{1}, \ldots, g_{n-1}\right) \varphi(a),
\end{aligned}
$$

where the right-hand side is an element of $\varphi(\mathcal{A})$ thanks to (51). Therefore, $\beta_{t^{-1}}\left(u\left(g_{1}, \ldots, g_{n}\right)\right) \varphi(\mathcal{A}) \subseteq \varphi(\mathcal{A})$, so, applying $\beta_{t}$, we obtain $u\left(g_{1}, \ldots, g_{n}\right) \beta_{t}(\varphi(\mathcal{A})) \subseteq$ $\beta_{t}(\varphi(\mathcal{A}))$. Similarly, $\beta_{t}(\varphi(\mathcal{A})) u\left(g_{1}, \ldots, g_{n}\right) \subseteq \beta_{t}(\varphi(\mathcal{A}))$, proving (50) in view of (42).

3.2. The construction of $w^{\prime}$. From now on we assume that $\mathcal{A}=\prod_{\lambda \in \Lambda} \mathcal{A}_{\lambda}$, where each $\mathcal{A}_{\lambda}$ is an indecomposable unital ring, called a block of $\mathcal{A}$. Our aim is to show that every $w \in Z_{\text {par }}^{n}(G, \mathcal{A})$ can be replaced by a more manageable $w^{\prime} \in Z_{\text {par }}^{n}(G, \mathcal{A})$ which will be used in the construction of $\widetilde{w}$ satisfying the conditions of Theorem 3.6.

As in [16], the identity $1_{\mathcal{A}_{\lambda}}$ of $\mathcal{A}_{\lambda}$ will be identified with an (indecomposable) central idempotent of $\mathcal{A}$, the block $\mathcal{A}_{\lambda}$ with the ideal $1_{\mathcal{A}_{\lambda}} \mathcal{A}$ of $\mathcal{A}$, and the canonical projection $\operatorname{pr}_{\lambda}: \mathcal{A} \rightarrow \mathcal{A}_{\lambda}$ with the multiplication by $1_{\mathcal{A}_{\lambda}}$ in $\mathcal{A}$. We write $a=$ $\prod_{\lambda \in \Lambda_{1}} a_{\lambda}$, where $\Lambda_{1} \subseteq \Lambda$ and $a_{\lambda} \in \mathcal{A}_{\lambda}$ for all $\lambda \in \Lambda_{1}$, if

$$
\operatorname{pr}_{\lambda}(a)= \begin{cases}a_{\lambda}, & \lambda \in \Lambda_{1}, \\ 0_{\mathcal{A}}, & \text { otherwise. }\end{cases}
$$

Thus, each idempotent $e$ of $\mathcal{A}$ is central and is of the form $\prod_{\lambda \in \Lambda_{1}} 1_{\mathcal{A}_{\lambda}}$, so that $e \mathcal{A}=\prod_{\lambda \in \Lambda_{1}} \mathcal{A}_{\lambda}$. Moreover, an isomorphism between two unital ideals $e \mathcal{A}$ and $f \mathcal{A}$ maps a block of $e \mathcal{A}$ onto a block of $f \mathcal{A}$ (see [16, Lemma 3.1]).

A unital partial action $\alpha$ of a group $G$ on $\mathcal{A}$ is called transitive, if for all $\lambda^{\prime}, \lambda^{\prime \prime} \in \Lambda$ there exists $x \in G$, such that $\mathcal{A}_{\lambda^{\prime}} \subseteq \mathcal{D}_{x^{-1}}$ and $\alpha_{x}\left(\mathcal{A}_{\lambda^{\prime}}\right)=\mathcal{A}_{\lambda^{\prime \prime}}$. As in [16], we fix $\lambda_{0} \in \Lambda$ and denote by $H$ the stabilizer of the block $\mathcal{A}_{\lambda_{0}}$, i.e. the subgroup

$$
H=\left\{x \in G \mid \mathcal{A}_{\lambda_{0}} \subseteq \mathcal{D}_{x^{-1}} \text { and } \alpha_{x}\left(\mathcal{A}_{\lambda_{0}}\right)=\mathcal{A}_{\lambda_{0}}\right\}
$$

of $G$. Let $\Lambda^{\prime}$ be a left transversal of $H$ in $G$ containing the identity element 1 of $G$. Then $\Lambda$ can be identified with a subset of $\Lambda^{\prime}$, namely, $\lambda_{0}$ is identified with 1 and

$$
\mathcal{A}_{g}=\alpha_{g}\left(\mathcal{A}_{1}\right) \text { for } g \in \Lambda \subseteq \Lambda^{\prime} .
$$

Given $x \in G$, denote by $\bar{x}$ the (unique) element of $\Lambda^{\prime}$, such that $x \in \bar{x} H$. We shall use the following easy fact throughout the text.

Lemma 3.7 (Lemma 5.1 from [11). Given $x \in G$ and $g \in \Lambda^{\prime}$, one has

(i) $g \in \Lambda \Leftrightarrow \mathcal{A}_{1} \subseteq \mathcal{D}_{g^{-1}}$;

(ii) if $g \in \Lambda$, then $\overline{x g} \in \Lambda \Leftrightarrow \mathcal{A}_{g} \subseteq \mathcal{D}_{x^{-1}}$, in which case $\alpha_{x}\left(\mathcal{A}_{g}\right)=\mathcal{A}_{\overline{x g}}$. 
It follows that

$$
\mathcal{A}_{g} \subseteq \mathcal{D}_{x} \Leftrightarrow \overline{x^{-1} g} \in \Lambda \Leftrightarrow \mathcal{A}_{1} \subseteq \mathcal{D}_{g^{-1} x}
$$

In particular,

$$
\mathcal{A}_{\bar{x}} \subseteq \mathcal{D}_{x}
$$

for all $x \in G$, such that $\bar{x} \in \Lambda$. by

As in [16], the definition of $w^{\prime}$ will involve the homomorphism $\theta_{g}: \mathcal{A} \rightarrow \mathcal{A}_{g}$ given

$$
\theta_{g}(a)=\alpha_{g}\left(\operatorname{pr}_{1}(a)\right)=\operatorname{pr}_{g}\left(\alpha_{g}\left(1_{g^{-1}} a\right)\right),
$$

where $g \in \Lambda$ and $a \in \mathcal{A}$. It follows that

$$
a=\prod_{g \in \Lambda} \theta_{g}\left(\alpha_{g^{-1}}\left(1_{g} a\right)\right)
$$

(see formula (31) from [16]). Another fact that we shall use:

$$
\theta_{g}(a)=\theta_{g}\left(1_{x} a\right)
$$

for any $x \in G$, such that $\mathcal{A}_{1} \subseteq \mathcal{D}_{x}$. In particular, this holds for $x \in H$ and for $x=g^{-1}$.

Lemma 3.8. Let $n>0$ and $w \in Z_{\text {par }}^{n}(G, \mathcal{A})$. Then

$$
\begin{aligned}
w\left(x_{1}, \ldots, x_{n}\right)= & 1_{\left(x_{1}, \ldots, x_{n}\right)} \prod_{g \in \Lambda} \theta_{g}\left[w\left(g^{-1} x_{1}, x_{2}, \ldots, x_{n}\right)\right. \\
& +\sum_{k=1}^{n-1}(-1)^{k} w\left(g^{-1}, x_{1}, \ldots, x_{k} x_{k+1}, \ldots, x_{n}\right) \\
& \left.+(-1)^{n} w\left(g^{-1}, x_{1}, \ldots, x_{n-1}\right)\right] .
\end{aligned}
$$

Proof. By (40) and (55)

$$
\begin{aligned}
w\left(x_{1}, \ldots, x_{n}\right)= & \prod_{g \in \Lambda} \theta_{g}\left(\alpha_{g^{-1}}\left(1_{g} w\left(x_{1}, \ldots, x_{n}\right)\right)\right) \\
= & \prod_{g \in \Lambda} \theta_{g}\left[1_{g^{-1}} w\left(g^{-1} x_{1}, x_{2}, \ldots, x_{n}\right)\right. \\
& +\sum_{k=1}^{n-1}(-1)^{k} 1_{g^{-1} x_{1} \ldots x_{k}} w\left(g^{-1}, x_{1}, \ldots, x_{k} x_{k+1}, \ldots, x_{n}\right) \\
& \left.+(-1)^{n} 1_{g^{-1} x_{1} \ldots x_{n}} w\left(g^{-1}, x_{1}, \ldots, x_{n-1}\right)\right] \\
= & \prod_{g \in \Lambda} \theta_{g}\left[1 _ { ( g ^ { - 1 } , x _ { 1 } , \ldots , x _ { n } ) } \left(w\left(g^{-1} x_{1}, x_{2}, \ldots, x_{n}\right)\right.\right. \\
& +\sum_{k=1}^{n-1}(-1)^{k} w\left(g^{-1}, x_{1}, \ldots, x_{k} x_{k+1}, \ldots, x_{n}\right) \\
& \left.\left.+(-1)^{n} w\left(g^{-1}, x_{1}, \ldots, x_{n-1}\right)\right)\right] .
\end{aligned}
$$

It remains to observe by (53) and (54) that

$\theta_{g}\left(1_{\left(g^{-1}, x_{1}, \ldots, x_{n}\right)}\right)=\operatorname{pr}_{g}\left(\alpha_{g}\left(1_{g^{-1}} 1_{\left(g^{-1}, x_{1}, \ldots, x_{n}\right)}\right)\right)=\operatorname{pr}_{g}\left(1_{g} 1_{\left(x_{1}, \ldots, x_{n}\right)}\right)=\operatorname{pr}_{g}\left(1_{\left(x_{1}, \ldots, x_{n}\right)}\right)$, 
GLOBALIZATION OF COHOMOLOGY IN THE SENSE OF ALVARES-ALVES-REDONDO 19

so that

$$
\prod_{g \in \Lambda} \theta_{g}\left(1_{\left(g^{-1}, x_{1}, \ldots, x_{n}\right)}\right)=\prod_{g \in \Lambda} \operatorname{pr}_{g}\left(1_{\left(x_{1}, \ldots, x_{n}\right)}\right)=1_{\left(x_{1}, \ldots, x_{n}\right)} .
$$

We recall here the notations from [16. We denote by $\eta$ the map $G \rightarrow H$ which sends $x \in G$ to $x^{-1} \bar{x} \in H$. For all $n>0$ and $g \in \Lambda^{\prime}$ we define $\eta_{n}^{g}: G^{n} \rightarrow H$ by

$$
\eta_{n}^{g}\left(x_{1}, \ldots, x_{n}\right)=\eta\left(x_{n}^{-1} \overline{x_{n-1}^{-1} \ldots x_{1}^{-1} g}\right)
$$

and $\tau_{n}^{g}: G^{n} \rightarrow H^{n}$ by

$$
\tau_{n}^{g}\left(x_{1}, \ldots, x_{n}\right)=\left(\eta_{1}^{g}\left(x_{1}\right), \eta_{2}^{g}\left(x_{1}, x_{2}\right), \ldots, \eta_{n}^{g}\left(x_{1}, \ldots, x_{n}\right)\right) .
$$

We notice here that

$$
\eta_{1}^{g}\left(x_{1}\right) \eta_{2}^{g}\left(x_{1}, x_{2}\right) \ldots \eta_{n}^{g}\left(x_{1}, \ldots, x_{n}\right)=\eta\left(x_{n}^{-1} \ldots x_{1}^{-1} g\right)=\eta_{1}^{g}\left(x_{1} \ldots x_{n}\right) .
$$

Furthermore, the functions $\sigma_{n, i}^{g}: G^{n} \rightarrow G^{n+1}, n>0,0 \leq i \leq n$, will be defined by

$$
\begin{aligned}
\sigma_{n, 0}^{g}\left(x_{1}, \ldots, x_{n}\right) & =\left(g^{-1}, x_{1}, \ldots, x_{n}\right), \\
\sigma_{n, i}^{g}\left(x_{1}, \ldots, x_{n}\right) & =\left(\tau_{i}^{g}\left(x_{1}, \ldots, x_{i}\right),\left(\overline{x_{i}^{-1} \ldots x_{1}^{-1} g}\right)^{-1}, x_{i+1}, \ldots, x_{n}\right), \quad 0<i<n, \\
\sigma_{n, n}^{g}\left(x_{1}, \ldots, x_{n}\right) & =\left(\tau_{n}^{g}\left(x_{1}, \ldots, x_{n}\right),\left(\overline{x_{n}^{-1} \ldots x_{1}^{-1} g}\right)^{-1}\right) .
\end{aligned}
$$

If $n=0$, then we set

$$
\sigma_{0,0}^{g}=g^{-1} \in G .
$$

Definition 3.9. Given $n>0$ and $w \in C_{p a r}^{n}(G, \mathcal{A})$, define $w^{\prime} \in C_{p a r}^{n}(G, \mathcal{A})$ and $\varepsilon \in C_{\text {par }}^{n-1}(G, \mathcal{A})$ by

$$
\begin{aligned}
w^{\prime}\left(x_{1}, \ldots, x_{n}\right) & =1_{\left(x_{1}, \ldots, x_{n}\right)} \prod_{g \in \Lambda} \theta_{g} \circ w \circ \tau_{n}^{g}\left(x_{1}, \ldots, x_{n}\right), \\
\varepsilon\left(x_{1}, \ldots, x_{n-1}\right) & =1_{\left(x_{1}, \ldots, x_{n-1}\right)} \prod_{g \in \Lambda} \theta_{g}\left(\sum_{i=0}^{n-1}(-1)^{i} w \circ \sigma_{n-1, i}^{g}\left(x_{1}, \ldots, x_{n-1}\right)\right) .
\end{aligned}
$$

When $n=1$, equality (67) should be understood as

$$
\varepsilon=\prod_{g \in \Lambda} \theta_{g}\left(w\left(g^{-1}\right)\right) \in \mathcal{A}
$$

We introduce here an additive analogue of the notation used in [16]:

$$
\begin{aligned}
\Sigma(l, m)= & \sum_{k=l, i=m}^{n-1}(-1)^{k+i} w \circ \sigma_{n-1, i}^{g}\left(x_{1}, \ldots, x_{k} x_{k+1}, \ldots, x_{n}\right) \\
& +\sum_{i=m}^{n-1}(-1)^{n+i} w \circ \sigma_{n-1, i}^{g}\left(x_{1}, \ldots, x_{n-1}\right),
\end{aligned}
$$

where $1 \leq l \leq n-1$ and $0 \leq m \leq n-1$ ( $n$ is assumed to be fixed). 
Lemma 3.10. For all $w \in Z_{\text {par }}^{1}(G, \mathcal{A})$ and $x \in G$ we have:

$$
\left(\delta^{0} \varepsilon\right)(x)-\alpha_{x}\left(1_{x^{-1}} \varepsilon\right)-w(x)=1_{x} \prod_{g \in \Lambda} \theta_{g}\left(-w\left(g^{-1} x\right)\right) .
$$

Moreover, for $n>1, w \in Z^{n}(G, \mathcal{A})$ and $x_{1}, \ldots, x_{n} \in G$ :

$$
\begin{aligned}
& \left(\delta^{n-1} \varepsilon\right)\left(x_{1}, \ldots, x_{n}\right)-\alpha_{x_{1}}\left(1_{x_{1}^{-1}} \varepsilon\left(x_{2}, \ldots, x_{n}\right)\right)-w\left(x_{1}, \ldots, x_{n}\right) \\
& =1_{\left(x_{1}, \ldots, x_{n}\right)} \prod_{g \in \Lambda} \theta_{g}\left(-w\left(g^{-1} x_{1}, x_{2}, \ldots, x_{n}\right)+\Sigma(1,1)\right) .
\end{aligned}
$$

Proof. By (39), (57) and (68) the left-hand side of (70) equals

$$
-1_{x} \varepsilon-w(x)=-1_{x} \prod_{g \in \Lambda} \theta_{g}\left(w\left(g^{-1}\right)\right)-1_{x} \prod_{g \in \Lambda} \theta_{g}\left(w\left(g^{-1} x\right)-w\left(g^{-1}\right)\right),
$$

proving (70).

Now by (40), (67) and (69)

$$
\begin{aligned}
& \left(\delta^{n-1} \varepsilon\right)\left(x_{1}, \ldots, x_{n}\right)-\alpha_{x_{1}}\left(1_{x_{1}^{-1}} \varepsilon\left(x_{2}, \ldots, x_{n}\right)\right) \\
& =\sum_{k=1}^{n-1}(-1)^{k} 1_{x_{1} \ldots x_{k}} \varepsilon\left(x_{1}, \ldots, x_{k} x_{k+1}, \ldots, x_{n}\right)+(-1)^{n} 1_{x_{1} \ldots x_{n}} \varepsilon\left(x_{1}, \ldots, x_{n-1}\right) \\
& =1_{\left(x_{1}, \ldots, x_{n}\right)} \prod_{g \in \Lambda} \theta_{g}\left(\sum_{k=1, i=0}^{n-1}(-1)^{k+i} w \circ \sigma_{n-1, i}^{g}\left(x_{1}, \ldots, x_{k} x_{k+1}, \ldots, x_{n}\right)\right) \\
& +1_{\left(x_{1}, \ldots, x_{n}\right)} \prod_{g \in \Lambda} \theta_{g}\left(\sum_{i=0}^{n-1}(-1)^{n+i} w \circ \sigma_{n-1, i}^{g}\left(x_{1}, \ldots, x_{n-1}\right)\right) \\
& =1_{\left(x_{1}, \ldots, x_{n}\right)} \prod_{g \in \Lambda} \theta_{g}(\Sigma(1,0)) .
\end{aligned}
$$

As in the proof of [16, Lemma 3.5], using (57) we conclude that the latter is

$$
w\left(x_{1}, \ldots, x_{n}\right)+1_{\left(x_{1}, \ldots, x_{n}\right)} \prod_{g \in \Lambda} \theta_{g}\left(-w\left(g^{-1} x_{1}, x_{2}, \ldots, x_{n}\right)+\Sigma(1,1)\right) .
$$

Lemma 3.11. For all $n>1, w \in Z_{\text {par }}^{n}(G, \mathcal{A}), g \in \Lambda$ and $x_{1}, \ldots, x_{n} \in G$ :

$$
\begin{aligned}
1_{\sigma_{n, 1}^{g}\left(x_{1}, \ldots, x_{n}\right)}\left(-w\left(g^{-1} x_{1}, x_{2}, \ldots, x_{n}\right)+\Sigma(1,1)\right) \\
=-\alpha_{\eta_{1}^{g}\left(x_{1}\right)}\left(1_{\eta_{1}^{g}\left(x_{1}\right)^{-1}} w \circ \sigma_{n-1,0}^{-x_{1}^{-1}}\left(x_{2}, \ldots, x_{n}\right)\right) \\
\quad+1_{\sigma_{n, 1}^{g}\left(x_{1}, \ldots, x_{n}\right)}\left(-w\left(\tau_{1}^{g}\left(x_{1}\right),\left(\overline{x_{1}^{-1} g}\right)^{-1} x_{2}, x_{3}, \ldots, x_{n}\right)+\Sigma(2,2)\right. \\
\left.\quad+\sum_{i=1}^{n-1}(-1)^{i+1} w \circ \sigma_{n-1, i}^{g}\left(x_{1} x_{2}, x_{3}, \ldots, x_{n}\right)\right) .
\end{aligned}
$$

Proof. By (36), (59), (60) and (63)

$$
\begin{aligned}
0 & =\left(\delta^{n} w\right) \circ \sigma_{n, 1}^{g}\left(x_{1}, \ldots, x_{n}\right) \\
& =\left(\delta^{n} w\right)\left(g^{-1} x_{1} \cdot \overline{x_{1}^{-1} g},\left(\overline{x_{1}^{-1} g}\right)^{-1}, x_{2}, \ldots, x_{n}\right)
\end{aligned}
$$


GLOBALIZATION OF COHOMOLOGY IN THE SENSE OF ALVARES-ALVES-REDONDO 21

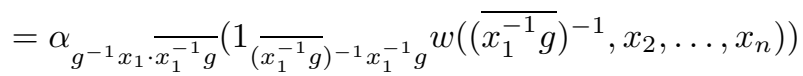

$$
\begin{aligned}
& -1_{g^{-1} x_{1} \cdot \overline{x_{1}^{-1} g}} w\left(g^{-1} x_{1}, x_{2}, \ldots, x_{n}\right) \\
& +1_{g^{-1} x_{1}} w\left(g^{-1} x_{1} \cdot \overline{x_{1}^{-1} g},\left(\overline{x_{1}^{-1} g}\right)^{-1} x_{2}, x_{3}, \ldots, x_{n}\right) \\
& +\sum_{k=2}^{n-1}(-1)^{k+1} 1_{g^{-1} x_{1} \ldots x_{k}} w\left(g^{-1} x_{1} \cdot \overline{x_{1}^{-1} g},\left(\overline{x_{1}^{-1} g}\right)^{-1}, x_{2}, \ldots, x_{k} x_{k+1}, \ldots x_{n}\right) \\
& +(-1)^{n+1} 1_{g^{-1} x_{1} \ldots x_{n}} w\left(g^{-1} x_{1} \cdot \overline{x_{1}^{-1} g},\left(\overline{x_{1}^{-1} g}\right)^{-1}, x_{2}, \ldots, x_{n-1}\right) .
\end{aligned}
$$

Therefore,

$$
\begin{aligned}
& -1_{\eta_{1}^{g}\left(x_{1}\right)} w\left(g^{-1} x_{1}, x_{2}, \ldots, x_{n}\right)=-\alpha_{\eta_{1}^{g}\left(x_{1}\right)}\left(1_{\eta_{1}^{g}\left(x_{1}\right)^{-1}} w \circ \sigma_{n-1,0}^{\overline{x_{1}^{-1} g}}\left(x_{2}, \ldots, x_{n}\right)\right) \\
& -1_{g^{-1} x_{1}} w\left(\tau_{1}^{g}\left(x_{1}\right),\left(\overline{x_{1}^{-1} g}\right)^{-1} x_{2}, x_{3}, \ldots, x_{n}\right) \\
& +\sum_{k=2}^{n-1}(-1)^{k} 1_{g^{-1} x_{1} \ldots x_{k}} w \circ \sigma_{n-1,1}^{g}\left(x_{1}, \ldots, x_{k} x_{k+1}, \ldots x_{n}\right) \\
& +(-1)^{n} 1_{g^{-1} x_{1} \ldots x_{n}} w \circ \sigma_{n-1,1}^{g}\left(x_{1}, \ldots, x_{n-1}\right) .
\end{aligned}
$$

Adding $\Sigma(1,1)$ and then multiplying both sides of the obtained equality by $1_{\sigma_{n, 1}^{g}\left(x_{1}, \ldots, x_{n}\right)}=$ $1_{\eta_{1}^{g}\left(x_{1}\right)} 1_{\left(g^{-1} x_{1}, x_{2}, \ldots, x_{n}\right)}$, we get (72) (for more details in the multiplicative case see the proof of [16, Lemma 3.6]).

Lemma 3.12. For all $1<j<n, w \in Z_{\text {par }}^{n}(G, \mathcal{A}), g \in \Lambda$ and $x_{1}, \ldots, x_{n} \in G$ :

$$
\begin{aligned}
1_{\sigma_{n, j}^{g}(}\left(x_{1}, \ldots, x_{n}\right) & \left(-w\left(\tau_{j-1}^{g}\left(x_{1}, \ldots, x_{j-1}\right),\left(\overline{x_{j-1}^{-1} \ldots x_{1}^{-1} g}\right)^{-1} x_{j}, x_{j+1}, \ldots, x_{n}\right)+\Sigma(j, j)\right) \\
= & (-1)^{j} \alpha_{\eta_{1}^{g}\left(x_{1}\right)}\left(1_{\eta_{1}^{g}\left(x_{1}\right)^{-1}} w \circ \sigma_{n-1, j-1}^{x_{1}^{-1} g}\left(x_{2}, \ldots, x_{n}\right)\right) \\
& +1_{\sigma_{n, j}^{g}\left(x_{1}, \ldots, x_{n}\right)}\left(-w\left(\tau_{j}^{g}\left(x_{1}, \ldots, x_{j}\right),\left(\overline{x_{j}^{-1} \ldots x_{1}^{-1} g}\right)^{-1} x_{j+1}, x_{j+2}, \ldots, x_{n}\right)+\Sigma(j+1, j+1)\right. \\
& +\sum_{i=j}^{n-1}(-1)^{i+j} w \circ \sigma_{n-1, i}^{g}\left(x_{1}, \ldots, x_{j} x_{j+1}, \ldots, x_{n}\right) \\
& \left.+\sum_{s=1}^{j-1}(-1)^{s+j} w \circ \sigma_{n-1, j-1}^{g}\left(x_{1}, \ldots, x_{s} x_{s+1}, \ldots, x_{n}\right)\right)
\end{aligned}
$$

(here by $\Sigma(n, n)$ we mean $\left.0_{\mathcal{A}}\right)$.

Proof. Our argument is analogous to that of the proof of Lemma 3.11 (for some technical details see also the proof of [16. Lemma 3.7]):

$$
\begin{aligned}
0= & \left(\delta^{n} w\right) \circ \sigma_{n, j}^{g}\left(x_{1}, \ldots, x_{n}\right) \\
= & \alpha_{\eta_{1}^{g}\left(x_{1}\right)}\left(1_{\eta_{1}^{g}\left(x_{1}\right)^{-1}} w \circ \sigma_{n-1, j-1}^{x_{1}^{-1} g}\left(x_{2}, \ldots, x_{n}\right)\right) \\
& +\sum_{s=1}^{j-1}(-1)^{s} 1_{\eta_{1}^{g}\left(x_{1} \ldots x_{s}\right)} w \circ \sigma_{n-1, j-1}^{g}\left(x_{1}, \ldots, x_{s} x_{s+1}, \ldots, x_{n}\right) \\
& +(-1)^{j} 1_{\eta_{1}^{g}\left(x_{1} \ldots x_{j}\right)} w\left(\tau_{j-1}^{g}\left(x_{1}, \ldots, x_{j-1}\right),\left(\overline{x_{j-1}^{-1} \ldots x_{1}^{-1}}\right)^{-1} x_{j}, x_{j+1}, \ldots, x_{n}\right) \\
& +(-1)^{j+1} 1_{g^{-1} x_{1} \ldots x_{j}} w\left(\tau_{j}^{g}\left(x_{1}, \ldots, x_{j}\right),\left(\overline{x_{j}^{-1} \ldots x_{1}^{-1} g}\right)^{-1} x_{j+1}, x_{j+2}, \ldots, x_{n}\right)
\end{aligned}
$$




$$
\begin{aligned}
& +\sum_{t=j+1}^{n-1}(-1)^{t+1} 1_{g^{-1} x_{1} \ldots x_{t}} w \circ \sigma_{n-1, j}^{g}\left(x_{1}, \ldots, x_{t} x_{t+1}, \ldots, x_{n}\right) \\
& +(-1)^{n+1} 1_{g^{-1} x_{1} \ldots x_{n}} w \circ \sigma_{n-1, j}^{g}\left(x_{1}, \ldots, x_{n-1}\right) .
\end{aligned}
$$

It follows that

$$
\begin{aligned}
& \left.-1_{\eta_{1}^{g}\left(x_{1} \ldots x_{j}\right)} w\left(\tau_{j-1}^{g}\left(x_{1}, \ldots, x_{j-1}\right), \overline{\left(x_{j-1}^{-1} \ldots x_{1}^{-1} g\right.}\right)^{-1} x_{j}, x_{j+1}, \ldots, x_{n}\right) \\
& =(-1)^{j} \alpha_{\eta_{1}^{g}\left(x_{1}\right)}\left(1_{\eta_{1}^{g}\left(x_{1}\right)^{-1}} w \circ \sigma_{n-1, j-1}^{x_{1}^{-1} g}\left(x_{2}, \ldots, x_{n}\right)\right) \\
& \quad+\sum_{s=1}^{j-1}(-1)^{s+j} 1_{\eta_{1}^{g}\left(x_{1} \ldots x_{s}\right)} w \circ \sigma_{n-1, j-1}^{g}\left(x_{1}, \ldots, x_{s} x_{s+1}, \ldots, x_{n}\right) \\
& \quad-1_{g^{-1} x_{1} \ldots x_{j}} w\left(\tau_{j}^{g}\left(x_{1}, \ldots, x_{j}\right),\left(\overline{x_{j}^{-1} \ldots x_{1}^{-1} g}\right)^{-1} x_{j+1}, x_{j+2}, \ldots, x_{n}\right) \\
& \quad+\sum_{t=j+1}^{n-1}(-1)^{t+j+1} 1_{g^{-1} x_{1} \ldots x_{t}} w \circ \sigma_{n-1, j}^{g}\left(x_{1}, \ldots, x_{t} x_{t+1}, \ldots, x_{n}\right) \\
& \quad+(-1)^{n+j+1} 1_{g^{-1} x_{1} \ldots x_{n}} w \circ \sigma_{n-1, j}^{g}\left(x_{1}, \ldots, x_{n-1}\right) .
\end{aligned}
$$

The addition of $\Sigma(j, j)$ followed by the multiplication of both sides by the idempotent

$$
1_{\sigma_{n, j}^{g}\left(x_{1}, \ldots, x_{n}\right)}=1_{\eta_{1}^{g}\left(x_{1}\right)} 1_{\eta_{1}^{g}\left(x_{1} x_{2}\right)} \ldots 1_{\eta_{1}^{g}\left(x_{1} \ldots x_{j}\right)} 1_{\left(g^{-1} x_{1} \ldots x_{j}, x_{j+1}, \ldots, x_{n}\right)}
$$

gives the desired equality (73).

Lemma 3.13. For all $w \in Z_{\text {par }}^{1}(G, \mathcal{A}), g \in \Lambda$ and $x \in G$ :

$$
-1_{\eta_{1}^{g}(x)} w\left(g^{-1} x\right)=-\alpha_{\eta_{1}^{g}(x)}\left(1_{\eta_{1}^{g}(x)^{-1}} w\left(\left(\overline{x^{-1} g}\right)^{-1}\right)\right)-1_{g^{-1} x} w \circ \tau_{1}^{g}(x) .
$$

Moreover, for all $n>1, w \in Z_{\text {par }}^{n}(G, \mathcal{A}), g \in \Lambda$ and $x_{1}, \ldots, x_{n} \in G$ :

$$
\begin{aligned}
& -1_{\eta_{1}^{g}\left(x_{1} \ldots x_{n}\right)} w\left(\tau_{n-1}^{g}\left(x_{1}, \ldots, x_{n-1}\right),\left(\overline{x_{n-1}^{-1} \ldots x_{1}^{-1} g}\right)^{-1} x_{n}\right) \\
& =(-1)^{n} \alpha_{\eta_{1}^{g}\left(x_{1}\right)}\left(1_{\eta_{1}^{g}\left(x_{1}\right)^{-1}} w \circ \sigma_{n-1, n-1}^{x_{1}^{-1} g}\right. \\
& \left.\quad+1_{\sigma_{n, n}^{g}\left(x_{1}, \ldots, x_{n}\right)}\left(\sum_{s=1}^{n-1}(-1)^{s+n} w \circ x_{n}\right)\right) \\
& \quad-w \circ \sigma_{n-1, n-1}^{g}\left(x_{1}, \ldots, x_{s} x_{s+1}, \ldots, x_{n}\right) \\
& \left.\left.\quad-\ldots, x_{n}\right)\right) .
\end{aligned}
$$

Proof. We first prove (74):

$$
\begin{aligned}
0 & =\left(\delta^{1} w\right)\left(\eta_{1}^{g}(x),\left(\overline{x^{-1} g}\right)^{-1}\right) \\
& =\alpha_{\eta_{1}^{g}(x)}\left(1_{\eta_{1}^{g}(x)^{-1}} w\left(\left(\overline{x^{-1} g}\right)^{-1}\right)\right)-1_{\eta_{1}^{g}(x)} w\left(g^{-1} x\right)+1_{g^{-1} x} w \circ \tau_{1}^{g}(x) .
\end{aligned}
$$

To get (75), write the following:

$$
\begin{aligned}
0= & \left(\delta^{n} w\right) \circ \sigma_{n, n}^{g}\left(x_{1}, \ldots, x_{n}\right) \\
= & \alpha_{\eta_{1}^{g}\left(x_{1}\right)}\left(1_{\eta_{1}^{g}\left(x_{1}\right)^{-1}} w \circ \sigma_{n-1, n-1}^{x_{1}^{-1} g}\left(x_{2}, \ldots, x_{n}\right)\right) \\
& +\sum_{s=1}^{n-1}(-1)^{s} 1_{\eta_{1}^{g}\left(x_{1} \ldots x_{s}\right)} w \circ \sigma_{n-1, n-1}^{g}\left(x_{1}, \ldots, x_{s} x_{s+1}, \ldots, x_{n}\right) \\
& +(-1)^{n} 1_{\eta_{1}^{g}\left(x_{1} \ldots x_{n}\right)} w\left(\tau_{n-1}^{g}\left(x_{1}, \ldots, x_{n-1}\right),\left(\overline{x_{n-1}^{-1} \ldots x_{1}^{-1} g}\right)^{-1} x_{n}\right)
\end{aligned}
$$


GLOBALIZATION OF COHOMOLOGY IN THE SENSE OF ALVARES-ALVES-REDONDO 23

$$
+(-1)^{n+1} 1_{g^{-1} x_{1} \ldots x_{n}} w \circ \tau_{n}^{g}\left(x_{1}, \ldots, x_{n}\right) .
$$

It remains to multiply both sides by $1_{\sigma_{n, n}^{g}\left(x_{1}, \ldots, x_{n}\right)}$.

Lemma 3.14. For all $n>0, w \in Z_{\text {par }}^{n}(G, \mathcal{A})$ and $x_{1}, \ldots, x_{n} \in G$ :

$$
\begin{aligned}
& \left(\delta^{n-1} \varepsilon\right)\left(x_{1}, \ldots, x_{n}\right)-\alpha_{x_{1}}\left(1_{x_{1}^{-1}} \varepsilon\left(x_{2}, \ldots, x_{n}\right)\right)-w\left(x_{1}, \ldots, x_{n}\right) \\
& =1_{\left(x_{1}, \ldots, x_{n}\right)} \prod_{g \in \Lambda} \theta_{g} \circ \alpha_{\eta_{1}^{g}\left(x_{1}\right)}\left(1_{\eta_{1}^{g}\left(x_{1}\right)^{-1}} \sum_{j=0}^{n-1}(-1)^{j+1} w \circ \overline{\sigma_{n-1, j}^{-1} g}\left(x_{2}, \ldots, x_{n}\right)\right) \\
& \quad-w^{\prime}\left(x_{1} \ldots, x_{n}\right) .
\end{aligned}
$$

Proof. Let $n=1$. Using (56), (58), (65), (66), (70) and (74), we have

$$
\begin{aligned}
\left(\delta^{0} \varepsilon\right)(x)-\alpha_{x}\left(1_{x^{-1}} \varepsilon\right)-w(x)= & 1_{x} \prod_{g \in \Lambda} \theta_{g}\left(-w\left(g^{-1} x\right)\right) \\
= & 1_{x} \prod_{g \in \Lambda} \theta_{g}\left(-1_{\eta_{1}^{g}(x)} w\left(g^{-1} x\right)\right) \\
= & \left.-1_{x} \prod_{g \in \Lambda} \theta_{g}\left(\alpha_{\eta_{1}^{g}(x)}\left(1_{\eta_{1}^{g}(x)^{-1}} w\left(\overline{\left(x^{-1} g\right.}\right)^{-1}\right)\right)\right) \\
& -1_{x} \prod_{g \in \Lambda} \theta_{g}\left(1_{g^{-1} x} w \circ \tau_{1}^{g}(x)\right) \\
= & -1_{x} \prod_{g \in \Lambda} \theta_{g} \circ \alpha_{\eta_{1}^{g}(x)}\left(1_{\eta_{1}^{g}(x)^{-1}} w\left(\overline{\sigma_{0,0}^{-1}}\right)\right) \\
& -w^{\prime}(x) .
\end{aligned}
$$

For $n>1$ we use equalities (172), (73) and (75) multiplied by the idempotent

$$
e=\prod_{i=1}^{n} 1_{\sigma_{n, i}^{g}\left(x_{1}, \ldots, x_{n}\right)}=1_{\left(g^{-1}, x_{1}, \ldots, x_{n}\right)} \prod_{i=1}^{n} 1_{\eta_{i}^{g}\left(x_{1}, \ldots, x_{i}\right)} .
$$

We have

$$
\begin{aligned}
& e\left(-w\left(g^{-1} x_{1}, x_{2}, \ldots, x_{n}\right)+\Sigma(1,1)\right) \\
& =e \alpha_{\eta_{1}^{g}\left(x_{1}\right)}\left(1_{\eta_{1}^{g}\left(x_{1}\right)^{-1}} \sum_{j=0}^{n-1}(-1)^{j+1} w \circ \sigma_{n-1, j}^{\overline{x_{1}^{-1} g}}\left(x_{2}, \ldots, x_{n}\right)\right) \\
& \quad-e\left(w \circ \tau_{n}^{g}\right)\left(x_{1}, \ldots, x_{n}\right) \\
& \quad+e \sum_{j=1}^{n-1} \sum_{i=j}^{n-1}(-1)^{i+j} w \circ \sigma_{n-1, i}^{g}\left(x_{1}, \ldots, x_{j} x_{j+1}, \ldots, x_{n}\right) \\
& \quad+e \sum_{j=2}^{n} \sum_{s=1}^{j-1}(-1)^{s+j} w \circ \sigma_{n-1, j-1}^{g}\left(x_{1}, \ldots, x_{s} x_{s+1}, \ldots, x_{n}\right) .
\end{aligned}
$$

Introducing $j^{\prime}=j-1$ in the sum (178), we rewrite it as

$$
\sum_{j^{\prime}=1}^{n-1} \sum_{s=1}^{j^{\prime}}(-1)^{s+j^{\prime}+1} w \circ \sigma_{n-1, j^{\prime}}^{g}\left(x_{1}, \ldots, x_{s} x_{s+1}, \ldots, x_{n}\right) .
$$


Switching the order of summation, we get

$$
\sum_{s=1}^{n-1} \sum_{j^{\prime}=s}^{n-1}(-1)^{s+j^{\prime}+1} w \circ \sigma_{n-1, j^{\prime}}^{g}\left(x_{1}, \ldots, x_{s} x_{s+1}, \ldots, x_{n}\right),
$$

which is the opposite of the sum (77). Hence,

$$
\begin{aligned}
e & \left(-w\left(g^{-1} x_{1}, x_{2}, \ldots, x_{n}\right)+\Sigma(1,1)\right) \\
= & e \alpha_{\eta_{1}^{g}\left(x_{1}\right)}\left(1_{\eta_{1}^{g}\left(x_{1}\right)^{-1}} \sum_{j=0}^{n-1}(-1)^{j+1} w \circ \sigma_{n-1, j}^{\overline{x_{1}^{-1}}}\left(x_{2}, \ldots, x_{n}\right)\right) \\
& -e\left(w \circ \tau_{n}^{g}\right)\left(x_{1}, \ldots, x_{n}\right) .
\end{aligned}
$$

Since $\eta_{i}^{g}\left(x_{1}, \ldots, x_{i}\right) \in H$ for all $i$, then after the application of $\theta_{g}$ to the both sides of (79), we may remove $\prod_{i=1}^{n} 1_{\eta_{i}^{g}\left(x_{1}, \ldots, x_{i}\right)}$ from $e$ by (56). Moreover, using (58), we obtain

$$
\begin{aligned}
& 1_{\left(x_{1}, \ldots, x_{n}\right)} \prod_{g \in \Lambda} \theta_{g}\left(-w\left(g^{-1} x_{1}, x_{2}, \ldots, x_{n}\right)+\Sigma(1,1)\right) \\
& =1_{\left(x_{1}, \ldots, x_{n}\right)} \prod_{g \in \Lambda} \theta_{g} \circ \alpha_{\eta_{1}^{g}\left(x_{1}\right)}\left(1_{\eta_{1}^{g}\left(x_{1}\right)^{-1}} \sum_{j=0}^{n-1}(-1)^{j+1} w \circ \sigma_{n-1, j}^{\overline{x_{1}^{-1} g}}\left(x_{2}, \ldots, x_{n}\right)\right) \\
& \quad-1_{\left(x_{1}, \ldots, x_{n}\right)} \prod_{g \in \Lambda} \theta_{g} \circ w \circ \tau_{n}^{g}\left(x_{1}, \ldots, x_{n}\right) .
\end{aligned}
$$

Then (76) follows by (66) and (71).

The following lemma is [16, Lemma 3.10].

Lemma 3.15. For all $x \in G$ and $a: \Lambda^{\prime} \rightarrow \mathcal{A}$ one has

$$
\alpha_{x}\left(1_{x^{-1}} \prod_{g \in \Lambda} \theta_{g}(a(g))\right)=1_{x} \prod_{g \in \Lambda} \theta_{g} \circ \alpha_{\eta_{1}^{g}(x)}\left(1_{\eta_{1}^{g}(x)^{-1}} a\left(\overline{x^{-1} g}\right)\right) .
$$

Lemma 3.16. For all $n>0, w \in Z_{\text {par }}^{n}(G, \mathcal{A})$ and $x_{1}, \ldots, x_{n} \in G$ :

$$
\begin{aligned}
& 1_{\left(x_{1}, \ldots, x_{n}\right)} \prod_{g \in \Lambda} \theta_{g} \circ \alpha_{\eta_{1}^{g}\left(x_{1}\right)}\left(1_{\eta_{1}^{g}\left(x_{1}\right)^{-1}} \sum_{j=0}^{n-1}(-1)^{j} w \circ \sigma_{n-1, j}^{\overline{x_{1}^{-1} g}}\left(x_{2}, \ldots, x_{n}\right)\right) \\
& =\alpha_{x_{1}}\left(1_{x_{1}^{-1}} \varepsilon\left(x_{2}, \ldots, x_{n}\right)\right) .
\end{aligned}
$$

Proof. Applying Lemma 3.15 with

$$
a(g)=\sum_{j=0}^{n-1}(-1)^{j} w \circ \sigma_{n-1, j}^{g}\left(x_{2}, \ldots, x_{n}\right)
$$

(where $n, w$ and $x_{2}, \ldots, x_{n}$ are fixed and $g \in \Lambda^{\prime}$ ), we see that the left-hand side of (81) equals

$$
1_{\left(x_{1} \ldots, x_{n}\right)} \alpha_{x_{1}}\left(1_{x_{1}^{-1}} \prod_{g \in \Lambda} \theta_{g}\left(\sum_{j=0}^{n-1}(-1)^{j} w \circ \sigma_{n-1, j}^{g}\left(x_{2}, \ldots, x_{n}\right)\right)\right) .
$$

Since $1_{\left(x_{1}, \ldots, x_{n}\right)}=\alpha_{x_{1}}\left(1_{x_{1}^{-1}} 1_{\left(x_{2}, \ldots, x_{n}\right)}\right)$, we obtain (81) by (67). 
As a consequence of Lemmas 3.14 and 3.16 we obtain the next.

Theorem 3.17. Let $n>0$ and $w \in Z_{\text {par }}^{n}(G, \mathcal{A})$. Then $w=\delta^{n-1} \varepsilon+w^{\prime}$. In particular, $w^{\prime} \in Z_{\text {par }}^{n}(G, \mathcal{A})$.

3.3. Existence and uniqueness of a globalization. Our aim in this section is to complete the construction of $\widetilde{w}$ satisfying (48) and (49). We start recalling the formulas from [16] which will be used here as well.

Lemma 3.18. Let $g \in \Lambda^{\prime}$. Then

$$
\begin{aligned}
\eta_{n}^{g}\left(x_{1}, \ldots, x_{n}\right) & =\eta_{n-1}^{\overline{x_{1}^{-1}}}\left(x_{2}, \ldots, x_{n}\right), n \geq 2, \\
\eta_{n}^{g}\left(x_{1}, \ldots, x_{i}, x_{i+1}, \ldots, x_{n}\right) & =\eta_{n-1}^{g}\left(x_{1}, \ldots, x_{i} x_{i+1}, \ldots, x_{n}\right), 1 \leq i \leq n-2, \\
\eta_{n}^{g}\left(x_{1}, \ldots, x_{n-1}, x_{n} x_{n+1}\right) & =\eta_{n}^{g}\left(x_{1}, \ldots, x_{n}\right) \eta_{n+1}^{g}\left(x_{1}, \ldots, x_{n+1}\right), n \geq 1 .
\end{aligned}
$$

Define $\widetilde{w^{\prime}}: G^{n} \rightarrow \mathcal{A}$ the same way as it was done in [16, i.e. by removing $1_{\left(x_{1}, \ldots, x_{n}\right)}$ from (66):

$$
\widetilde{w^{\prime}}\left(x_{1}, \ldots, x_{n}\right)=\prod_{g \in \Lambda} \theta_{g} \circ w \circ \tau_{n}^{g}\left(x_{1}, \ldots, x_{n}\right) .
$$

Lemma 3.19. Let $n>0, w \in Z_{p a r}^{n}(G, A)$ and $x_{1}, \ldots, x_{n} \in G$. Then

$$
\tilde{\delta}^{n} \widetilde{w^{\prime}}=0 \text {. }
$$

Proof. Using (47), (80) and (85), we rewrite the left-hand side of (86) as follows

$$
\begin{aligned}
1_{x_{1}} & \prod_{g \in \Lambda} \theta_{g} \circ \alpha_{\eta_{1}^{g}\left(x_{1}\right)}\left(1_{\eta_{1}^{g}\left(x_{1}\right)^{-1}} w \circ \tau_{n}^{\overline{x_{1}^{-1} g}}\left(x_{2}, \ldots, x_{n+1}\right)\right) \\
& +1_{x_{1}} \prod_{g \in \Lambda} \theta_{g}\left(\sum_{i=1}^{n}(-1)^{i} w \circ \tau_{n}^{g}\left(x_{1}, \ldots, x_{i} x_{i+1}, \ldots, x_{n+1}\right)\right) \\
& +1_{x_{1}} \prod_{g \in \Lambda} \theta_{g}\left((-1)^{n+1} w \circ \tau_{n}^{g}\left(x_{1}, \ldots, x_{n}\right)\right) .
\end{aligned}
$$

Notice by (56) that one can multiply the $i$-th term of the sum in (87) by $1_{\eta_{1}^{g}\left(x_{1} \ldots x_{i}\right)}$. And similarly, the argument of $\theta_{g}$ in (88) can be multiplied by $1_{\eta_{1}^{g}\left(x_{1} \ldots x_{n}\right)}$ without changing the value of the map. Hence, for (86), it suffices to prove

$$
\begin{aligned}
0= & \alpha_{\eta_{1}^{g}\left(x_{1}\right)}\left(1_{\eta_{1}^{g}\left(x_{1}\right)^{-1}} w \circ \overline{\tau_{n}^{x_{1}^{-1} g}}\left(x_{2}, \ldots, x_{n+1}\right)\right) \\
& +\sum_{i=1}^{n}(-1)^{i} 1_{\eta_{1}^{g}\left(x_{1} \ldots x_{i}\right)} w \circ \tau_{n}^{g}\left(x_{1}, \ldots, x_{i} x_{i+1}, \ldots, x_{n+1}\right) \\
& +(-1)^{n+1} 1_{\eta_{1}^{g}\left(x_{1} \ldots x_{n}\right)} w \circ \tau_{n}^{g}\left(x_{1}, \ldots, x_{n}\right) .
\end{aligned}
$$

As in the proof of [16, Lemma 4.2], one shows using (82) (84) that (89) is an expansion of the cocycle identity

$$
\left(\delta^{n} w\right) \circ \tau_{n+1}^{g}\left(x_{1}, \ldots, x_{n+1}\right)=0 .
$$


Now, for all $n>0$ and $x_{1}, \ldots, x_{n} \in G$, define $\widetilde{w}: G^{n} \rightarrow \mathcal{A}$ as the sum

$$
\widetilde{w}=\widetilde{w^{\prime}}+\hat{\delta}^{n-1} \varepsilon,
$$

where

$$
\begin{aligned}
\left(\hat{\delta}^{n-1} \varepsilon\right)\left(x_{1}, \ldots, x_{n}\right)= & \alpha_{x_{1}}\left(1_{x_{1}^{-1}} \varepsilon\left(x_{2}, \ldots, x_{n}\right)\right) \\
& +\sum_{i=1}^{n-1}(-1)^{i} \varepsilon\left(x_{1}, \ldots, x_{i} x_{i+1}, \ldots, x_{n}\right) \\
& +(-1)^{n} \varepsilon\left(x_{1}, \ldots, x_{n-1}\right) .
\end{aligned}
$$

The existence of a globalization is established in the following theorem.

Theorem 3.20. Let $\mathcal{A}$ be a direct product of indecomposable unital rings and $\alpha=$ $\left\{\alpha_{g}: \mathcal{D}_{g^{-1}} \rightarrow \mathcal{D}_{g} \mid g \in G\right\}$ a (non-necessarily transitive) unital partial action of $G$ on $\mathcal{A}$. Then for any $n \geq 0$ each cocycle $w \in Z_{\text {par }}^{n}(G, \mathcal{A})$ with values in the induced $K_{\text {par }}(G)$-module is globalizable.

Proof. The case $n=0$ is the existence part of Lemma 3.4, so let $n>0$. As in the proof of [16. Theorem 6.3], it is enough to consider transitive $\alpha$. The map $\widetilde{w}: G^{n} \rightarrow \mathcal{A}$ defined in (90) satisfies (49), as

$$
\begin{aligned}
1_{\left(x_{1}, \ldots, x_{n}\right)} \widetilde{w}\left(x_{1}, \ldots, x_{n}\right) & =1_{\left(x_{1}, \ldots, x_{n}\right)} \widetilde{w^{\prime}}\left(x_{1}, \ldots, x_{n}\right)+1_{\left(x_{1}, \ldots, x_{n}\right)}\left(\hat{\delta}^{n-1} \varepsilon\right)\left(x_{1}, \ldots, x_{n}\right) \\
& =w^{\prime}\left(x_{1}, \ldots, x_{n}\right)+\left(\delta^{n-1} \varepsilon\right)\left(x_{1}, \ldots, x_{n}\right) \\
& =w\left(x_{1}, \ldots, x_{n}\right) .
\end{aligned}
$$

for all $x_{1}, \ldots, x_{n} \in G$ by (40), (47), (66) and (85) and Theorem 3.17

To apply Theorem 3.6, it remains to prove (48). Observe by (90) that

$$
\left(\tilde{\delta}^{n} \widetilde{w}\right)\left(x_{1}, \ldots, x_{n+1}\right)=\left(\tilde{\delta}^{n} \widetilde{w^{\prime}}\right)\left(x_{1}, \ldots, x_{n+1}\right)+\left(\tilde{\delta}^{n} \hat{\delta}^{n-1} \varepsilon\right)\left(x_{1}, \ldots, x_{n+1}\right),
$$

where the first summand is zero by Lemma 3.19 Comparing $\tilde{\delta}^{n} \hat{\delta}^{n-1} \varepsilon$ with the classical $\delta^{n} \delta^{n-1} \varepsilon$, we see that the difference is that instead of a global action one has $\alpha$, and all the terms to which $\alpha$ is not applied are multiplied by $1_{x_{1}}$. The latter terms cancel, and the remaining ones are

$$
\alpha_{x_{1}}\left(1_{x_{1}^{-1}}\left(\hat{\delta}^{n-1} \varepsilon\right)\left(x_{2}, \ldots, x_{n+1}\right)\right)
$$

and the first summands in each

$$
\begin{aligned}
& (-1)^{i} 1_{x_{1}}\left(\hat{\delta}^{n-1} \varepsilon\right)\left(x_{1}, \ldots, x_{i} x_{i+1}, \ldots, x_{n+1}\right), 1 \leq i \leq n, \\
& (-1)^{n+1} 1_{x_{1}}\left(\hat{\delta}^{n-1} \varepsilon\right)\left(x_{1}, \ldots, x_{n}\right) .
\end{aligned}
$$

The terms of the expansion of (92) are

$$
\begin{aligned}
& \alpha_{x_{1}}\left(1_{x_{1}^{-1}} \alpha_{x_{2}}\left(1_{x_{2}^{-1}} \varepsilon\left(x_{3}, \ldots, x_{n+1}\right)\right)\right), \\
& \alpha_{x_{1}}\left((-1)^{i-1} 1_{x_{1}^{-1}} \varepsilon\left(x_{2}, \ldots, x_{i} x_{i+1}, \ldots, x_{n+1}\right)\right), 2 \leq i \leq n, \\
& (-1)^{n} \alpha_{x_{1}}\left(1_{x_{1}^{-1}} \varepsilon\left(x_{2}, \ldots, x_{n}\right)\right),
\end{aligned}
$$

while the first summands in (93) and (94) are

$$
-1_{x_{1}} \alpha_{x_{1} x_{2}}\left(1_{x_{2}^{-1} x_{1}^{-1}} \varepsilon\left(x_{3}, \ldots, x_{n+1}\right)\right)
$$


GLOBALIZATION OF COHOMOLOGY IN THE SENSE OF ALVARES-ALVES-REDONDO 27

$$
\begin{aligned}
& (-1)^{i} \alpha_{x_{1}}\left(1_{x_{1}^{-1}} \varepsilon\left(x_{2}, \ldots, x_{i} x_{i+1}, \ldots, x_{n+1}\right)\right), 2 \leq i \leq n, \\
& (-1)^{n+1} \alpha_{x_{1}}\left(1_{x_{1}^{-1}} \varepsilon\left(x_{2}, \ldots, x_{n}\right)\right) .
\end{aligned}
$$

Thus, (95) cancels with (98), (96) with (99), and (97) with (100).

We recall the following result from [16.

Proposition 3.21. Let $\mathcal{A}$ be a direct product $\prod_{g \in \Lambda} \mathcal{A}_{g}$ of indecomposable unital rings, $\alpha$ a transitive unital partial action of $G$ on $\mathcal{A}$ and $(\beta, \mathcal{B})$ an enveloping action of $(\alpha, \mathcal{A})$ with $\mathcal{A} \subseteq \mathcal{B}$. Then $\mathcal{B}$ embeds as an ideal into $\prod_{g \in \Lambda^{\prime}} \mathcal{A}_{g}$, where $\mathcal{A}_{g}$ denotes the ideal $\beta_{g}\left(\mathcal{A}_{1}\right)$ in $\mathcal{B}$. Moreover, $\mathcal{M}(\mathcal{B}) \cong \prod_{g \in \Lambda^{\prime}} \mathcal{A}_{g}$, and $\beta^{*}$ is transitive, when seen as a partial action of $G$ on $\prod_{g \in \Lambda^{\prime}} \mathcal{A}_{g}$.

This permits us to obtain the uniqueness of a globalization.

Theorem 3.22. Let $\mathcal{A}$ be a direct product $\prod_{g \in \Lambda} \mathcal{A}_{g}$ of indecomposable unital rings, $\alpha$ a unital partial action of $G$ on $\mathcal{A}$ and $w_{i} \in Z_{\text {par }}^{n}(G, \mathcal{A}), i=1,2(n>0)$. Suppose that $(\beta, \mathcal{B})$ is an enveloping action of $(\alpha, \mathcal{A})$ and $u_{i} \in Z^{n}(G, \mathcal{M}(\mathcal{B}))$ is a globalization of $w_{i}, i=1,2$. If $w_{1}$ is cohomologous to $w_{2}$, then $u_{1}$ is cohomologous to $u_{2}$. In particular, any two globalizations of the same partial n-cocycle are cohomologous.

Proof. As in the proof of [16. Theorem 5.3], we consider only the transitive case and assume, without loss of generality, that $\mathcal{M}(\mathcal{B})=\prod_{g \in \Lambda^{\prime}} \mathcal{A}_{g} \supseteq \mathcal{A}$. Then we define the homomorphism $\vartheta_{g}: \mathcal{M}(\mathcal{B}) \rightarrow \mathcal{M}(\mathcal{B})$ by

$$
\vartheta_{g}=\beta_{g} \circ \mathrm{pr}_{1}
$$

and $u_{i}^{\prime} \in C^{n}(G, \mathcal{M}(\mathcal{B}))$ by

$$
u_{i}^{\prime}\left(x_{1}, \ldots, x_{n}\right)=\prod_{g \in \Lambda^{\prime}} \vartheta_{g} \circ u_{i} \circ \tau_{n}^{g}\left(x_{1}, \ldots, x_{n}\right), i=1,2 .
$$

We infer that $u_{i}^{\prime} \in Z^{n}(G, \mathcal{M}(\mathcal{B}))$ and $u_{i}$ is cohomologous to $u_{i}^{\prime}, i=1,2$, because the definition of $u_{i}^{\prime}$ is totally analogous to that of $w^{\prime}$ (compare (102) with (66) and (101) with (54), see also Theorem 3.17).

Suppose that $w_{1}$ is cohomologous to $w_{2}$. To prove that $u_{1}$ is cohomologous to $u_{2}$, it is enough to establish the cohomological equivalence of $u_{1}^{\prime}$ and $u_{2}^{\prime}$. Observe as in the proof of [16. Theorem 5.3] that

$$
u_{i}^{\prime}\left(x_{1}, \ldots, x_{n}\right)=\prod_{g \in \Lambda^{\prime}} \vartheta_{g} \circ w_{i} \circ \tau_{n}^{g}\left(x_{1}, \ldots, x_{n}\right), i=1,2 .
$$

If $w_{2}=w_{1}+\delta^{n-1} \xi$ for some $\xi \in C_{\text {par }}^{n-1}(G, \mathcal{A})$, then by (103) one readily gets $u_{2}^{\prime}=u_{1}^{\prime}+\left(\delta^{n-1} \xi\right)^{\prime}$, where

$$
\left(\delta^{n-1} \xi\right)^{\prime}\left(x_{1}, \ldots, x_{n}\right)=\prod_{g \in \Lambda^{\prime}} \vartheta_{g} \circ\left(\delta^{n-1} \xi\right) \circ \tau_{n}^{g}\left(x_{1}, \ldots, x_{n}\right) .
$$

It is enough to prove that

$$
\left(\delta^{n-1} \xi\right)^{\prime}=\delta^{n-1} \xi^{\prime}
$$

where

$$
\xi^{\prime}\left(x_{1}, \ldots, x_{n-1}\right)=\prod_{g \in \Lambda^{\prime}} \vartheta_{g} \circ \xi \circ \tau_{n-1}^{g}\left(x_{1}, \ldots, x_{n-1}\right) .
$$


Expanding $\left(\delta^{n-1} \xi\right)^{\prime}\left(x_{1}, \ldots, x_{n}\right)$, we see that the idempotents $1_{\eta_{i}^{g}\left(x_{1} \ldots x_{i}\right)}$ which appear in the expansion may be removed, because the analogue of (56) holds for $\vartheta_{g}$ too (compare (101) with (54)). Thus, due to the fact that $\vartheta_{g}$ is a homomorphism, (104) reduces to

$$
\begin{aligned}
& \beta_{x_{1}}\left(\prod_{g \in \Lambda^{\prime}} \vartheta_{g} \circ \xi \circ \tau_{n-1}^{g}\left(x_{2}, \ldots, x_{n}\right)\right) \\
& =\prod_{g \in \Lambda^{\prime}} \vartheta_{g} \circ \beta_{\eta^{g}\left(x_{1}\right)} \circ \xi\left(\eta_{2}^{g}\left(x_{1}, x_{2}\right), \ldots, \eta_{n}^{g}\left(x_{1}, \ldots, x_{n}\right)\right) \\
& =\prod_{g \in \Lambda^{\prime}} \vartheta_{g} \circ \beta_{\eta^{g}\left(x_{1}\right)} \circ \xi \circ \overline{\tau_{n-1}^{-1} g}\left(x_{2}, \ldots, x_{n}\right),
\end{aligned}
$$

which follows from the global version of Lemma 3.15

Corollary 3.23. Let $\mathcal{A}$ be a direct product $\prod_{g \in \Lambda} \mathcal{A}_{g}$ of indecomposable unital rings, $\alpha$ a partial action of $G$ on $\mathcal{A}$ and $(\beta, \mathcal{B})$ an enveloping action of $(\alpha, \mathcal{A})$. Then $H_{\text {par }}^{n}(G, \mathcal{A})$ is isomorphic to the classical cohomology group $H^{n}(G, \mathcal{M}(\mathcal{B}))$.

Indeed, we know by Proposition 3.2 that for all $n \geq 0$ there is a homomorphism $H^{n}(G, \mathcal{M}(\mathcal{B})) \rightarrow H_{\text {par }}^{n}(G, \mathcal{A})$ coming from the restriction map. By Theorems 3.20 and 3.22 this homomorphism is invertible, when $n>0$. The case $n=0$ is Lemma 3.4

\section{ACKNOWLEDGMENTS}

This work was partially supported by CNPq of Brazil (Proc. 307873/2017-0, 404649/2018-1), FAPESP of Brazil (Proc. 2015/09162-9), MINECO (MTM201677445-P), Fundación Séneca of Spain and Fundação para a Ciência e a Tecnologia (Portuguese Foundation for Science and Technology) through the project PTDC/MAT-PUR/31174/2017. The first two authors would also like to thank the Department of Mathematics of the University of Murcia for its warm hospitality during their visits.

\section{REFERENCES}

[1] Abadie, F. Enveloping actions and Takai duality for partial actions. J. Funct. Anal. 197, 1 (2003), 14-67.

[2] Alvares, E. R., Alves, M. M., and Redondo, M. J. Cohomology of partial smash products. J. Algebra 482 (2017), 204-223.

[3] Alves, M. M., Dokuchaev, M., And Kochloukova, D. Homology and cohomology via the partial group algebra. Preprint.

[4] Batista, E., Mortari, A. D. M., and Teixeira, M. M. Cohomology for partial actions of Hopf algebras. J. Algebra 528 (2019), 339-380.

[5] Caenepeel, S., and Janssen, K. Partial (co)actions of Hopf algebras and partial Hopf-Galois theory. Comm. Algebra 36, 8 (2008), 2923-2946.

[6] Dokuchaev, M. Recent developments around partial actions. São Paulo J. Math. Sci. 13, 1 (2019), 195-247.

[7] Dokuchaev, M., Del Río, Á., And Simón, J. J. Globalizations of partial actions on nonunital rings. Proc. Am. Math. Soc. 135, 2 (2007), 343-352.

[8] Dokuchaev, M., and Exel, R. Associativity of crossed products by partial actions, enveloping actions and partial representations. Trans. Amer. Math. Soc. 357, 5 (2005), 1931-1952.

[9] Dokuchaev, M., Exel, R., And Piccione, P. Partial representations and partial group algebras. J. Algebra 226, 1 (2000), 251-268. 
[10] Dokuchaev, M., Exel, R., and Simón, J. J. Crossed products by twisted partial actions and graded algebras. J. Algebra 320, 8 (2008), 3278-3310.

[11] Dokuchaev, M., Exel, R., And Simón, J. J. Globalization of twisted partial actions. Trans. Amer. Math. Soc. 362, 8 (2010), 4137-4160.

[12] Dokuchaev, M., Ferrero, M., and Paques, A. Partial Actions and Galois Theory. J. Pure Appl. Algebra 208, 1 (2007), 77-87.

[13] Dokuchaev, M., and Khrypchenko, M. Partial cohomology of groups. J. Algebra 427 (2015), 142-182.

[14] Dokuchaev, M., and Khrypchenko, M. Twisted partial actions and extensions of semilattices of groups by groups. Int. J. Algebra Comput. 27, 7 (2017), 887-933.

[15] Dokuchaev, M., and Khrypchenko, M. Partial cohomology of groups and extensions of semilattices of abelian groups. J. Pure Appl. Algebra 222 (2018), 2897-2930.

[16] Dokuchaev, M., Khrypchenko, M., And Simón, J. J. Globalization of partial cohomology of groups. arXiv:1706.02546 (2017).

[17] Dokuchaev, M., And Novikov, B. Partial projective representations and partial actions. J. Pure Appl. Algebra 214, 3 (2010), 251-268.

[18] Dokuchaev, M., And Novikov, B. Partial projective representations and partial actions II. J. Pure Appl. Algebra 216, 2 (2012), 438-455.

[19] Dokuchaev, M., Novikov, B., And Pinedo, H. The partial Schur Multiplier of a group. J. Algebra 392 (2013), 199-225.

[20] Dokuchaev, M., Paques, A., And Pinedo, H. Partial galois cohomology and related homomorphisms. Quarterly J. Math. 70, 2 (2019), 737-766.

[21] Dokuchaev, M., Paques, A., Pinedo, H., And Rocha, I. Partial generalized crossed products and a seven-term exact sequence. arXiv:1908.05820 (2019).

[22] Dokuchaev, M., and Sambonet, N. Schur's theory for partial projective representations. Israel J. Math. 232, 1 (2019), 373-399.

[23] Dokuchaev, M., and Zhukavets, N. On finite degree partial representations of groups. $J$. Algebra 274, 1 (2004), 309-334.

[24] Exel, R. Twisted partial actions: a classification of regular $C^{*}$-algebraic bundles. Proc. London Math. Soc. 74, 3 (1997), 417-443.

[25] Exel, R. Partial actions of groups and actions of inverse semigroups. Proc. Amer. Math. Soc. 126, 12 (1998), 3481-3494.

[26] Kellendonk, J., and Lawson, M. V. Partial actions of groups. Internat. J. Algebra Comput. 14, 1 (2004), 87-114.

[27] Kennedy, M., and Schafhauser, C. Noncommutative boundaries and the ideal structure of reduced crossed products. To appear in Duke Math. J. doi:10.1215/00127094-2019-0032 (2019).

[28] Maclane, S. Homology. Springer-Verlag, Berlin-Guttingen-Heidelberg, 1963.

[29] Passman, D. S. A course in ring theory. The Wadsworth \& Brooks/Cole Mathematics Series. Wadsworth \& Brooks/Cole Advanced Books \& Software, Pacific Grove, CA, 1991.

Insituto de Matemática e Estatística, Universidade de São Paulo, Rua do Matão, 1010, SÃo Paulo, SP, CEP: 05508-090, Brazil

E-mail address: dokucha@gmail.com

Departamento de Matemática, Universidade Federal de Santa Catarina, Campus Reitor João David Ferreira lima, Florianópolis, SC, CEP: 88040-900, Brazil and Centro de Matemática e Aplicações, Faculdade de Ciências e Tecnologia, Universidade Nova De Lisboa, 2829-516 Caparica, Portugal

E-mail address: nskhripchenko@gmail.com

Departamento de Matemáticas, Universidad de Murcia, 30071 Murcia, Spain

E-mail address: jsimon@um.es 